\title{
Gravitational instability of slowly rotating isothermal spheres
}

\author{
P. H. Chavanis ${ }^{\star}$
}

\author{
Laboratoire de Physique Quantique, Université Paul Sabatier, 118 route de Narbonne, 31062 Toulouse, France
}

Received 15 April 2002 / Accepted 12 September 2002

\begin{abstract}
We discuss the statistical mechanics of rotating self-gravitating systems by allowing properly for the conservation of angular momentum. We study analytically the case of slowly rotating isothermal spheres by expanding the solutions of the Boltzmann-Poisson equation in a series of Legendre polynomials, adapting the procedure introduced by Chandrasekhar (1933) for distorted polytropes. We show how the classical spiral of Lynden-Bell \& Wood (1967) in the temperature-energy plane is deformed by rotation. We find that gravitational instability occurs sooner in the microcanonical ensemble and later in the canonical ensemble. According to standard turning point arguments, the onset of the collapse coincides with the minimum energy or minimum temperature state in the series of equilibria. Interestingly, it happens to be close to the point of maximum flattening. We generalize the singular isothermal solution to the case of a slowly rotating configuration. We also consider slowly rotating configurations of the self-gravitating Fermi gas at non-zero temperature.
\end{abstract}

Key words. hydrodynamics - instabilities - stars: formation

\section{Introduction}

Recently, the statistical mechanics of self-gravitating systems has attracted considerable attention (Chavanis et al. 1996; Chavanis \& Sommeria 1998; de Vega et al. 1998; Youngkins \& Miller 2000; Follana \& Laliena 2000; Semelin et al. 2001; Cerruti-Sola et al. 2001; Ispolatov \& Cohen 2001; Chavanis 2002a; Taruya \& Sakagami 2002; de Vega \& Sanchez 2002; Huber \& Pfenniger 2002...). This topic was introduced in the 1960s by Antonov (1962) and Lynden-Bell \& Wood (1968) and further developed by Hertel \& Thirring (1971), Horwitz \& Katz (1978), Katz (1978) and Padmanabhan (1989) among others (see a complete list of references in the review of Padmanabhan 1990). These authors pointed out the particularity of self-gravitating systems to possess negative specific heats. They showed that this strange property is responsible for the inequivalence of statistical ensembles (microcanoni$\mathrm{cal} /$ canonical) and the occurence of giant phase transitions associated with gravitational collapse. For a long time, these topics were only discussed in the astrophysical literature and were considered as a curiosity (not to say a fallacy) by statistical mechanicians. The situation is changing lately as these properties are re-discovered for other physical systems with longrange interactions which can be studied in the laboratory (see, e.g., Gross 2001). For that reason, the statistical mechanics of self-gravitating systems comes back to fashion with new perspectives.

The statistical mechanics of self-gravitating systems is far from being completely understood and rests on simplifying idealizations. The first idealization is to enclose the system within

^ e-mail: chavanis@irsamc2.ups-tlse.fr a box so as to prevent evaporation. It is only under this condition (or by introducing more realistic truncated models) that a rigorous statistical mechanics of self-gravitating systems can be carried out. Second, for most astrophysical systems, the relaxation time by two-body encounters is much larger than the age of the universe so that a more subtle, collisionless, relaxation must be advocated to explain the structure of galaxies. This is the concept of violent relaxation formalized by LyndenBell in 1967. Then, it is implicitly assumed that the relaxation towards statistical equilibrium proceeds to completion, which is not necessarily the case in reality. Indeed, it is possible that the relaxation stops before the maximum entropy state is attained (see Lynden-Bell 1967; Tremaine et al. 1987; Chavanis et al. 1996). This problem of incomplete relaxation must be approached with extensive numerical simulations. Of course, this program was started long ago (e.g., van Albada 1982) but only recently are $N$-body simulations carefully compared with the predictions of the statistical mechanics approach (Cerruti-Sola et al. 2001; Huber \& Pfenniger 2002) with variable success.

The statistical equilibrium of a non-rotating classical gas enclosed within a box was first investigated by Antonov (1962). He worked in the microcanonical ensemble and found that thermodynamical equilibrium exists only above a critical energy $E_{\min }=-0.335 G M^{2} / R$. Below that energy, the system is expected to collapse and overheat; this is the so-called "gravothermal catastrophe" (Lynden-Bell \& Wood 1968). An isothermal collapse also occurs below a critical temperature $T_{\min }=\frac{G M m}{2.52 R}$ in the canonical ensemble. This thermodynamical instability is closely related to the dynamical Jeans instability (Semelin et al. 2001; Chavanis 2002a). In a recent series of papers, we considered some extensions of the Antonov 
problem to the case of self-gravitating fermions (Chavanis \& Sommeria 1998; Chavanis 2002c), special and general relativity (Chavanis 2002b) and confined polytropes (Chavanis 2002d). We also introduced a simple dynamical model of Brownian particles in gravitational interaction (Chavanis et al. 2002; Sire \& Chavanis 2002). By adding by hands a friction and a noise, we force the system to increase entropy continuously, thereby avoiding the problem of incomplete relaxation. This model can be used to test precisely some ideas of statistical mechanics (inequivalence of ensembles, phase transitions, gravitational instabilities, basin of attraction,...) and is sufficiently simple to allow for a thorough analytical investigation of the collapse regime when an equilibrium state does not exist.

In all these studies, the system is assumed to be nonrotating so that the conservation of angular momentum is trivially satisfied. The object of the present paper is to extend the statistical mechanics approach to the case of rotating self-gravitating systems. This problem has been considered previously by Lagoute \& Longaretti (1996), Laliena (1999), Lynden-Bell (2000) and Fliegans \& Gross (2002) with different types of models. Clearly, the most interesting situation is the case of rapidly rotating systems since a wide variety of structures can emerge as maximum entropy states (Votyakov et al. 2002). However, we shall restrict ourselves in the present paper to the case of slowly rotating systems for which the problem can be tackled analytically. We shall adapt to the case of isothermal spheres the classical procedure developed by Chandrasekhar (1933) for distorted polytropes, i.e. we shall expand the solutions of the Boltzmann-Poisson equation in terms of Legendre polynomials. A similar procedure was performed by Lagoute \& Longaretti (1996) for rotating globular clusters subject to tidal forces and described by an extended MichieKing model. We believe that it is useful to consider the case of a gas enclosed within a spherical box so as to make a clear connexion with the Antonov model when the rotation is set to zero. In particular, we shall derive the expression of the thermodynamical parameters for slowly rotating isothermal spheres and show how the classical spiral of Lynden-Bell \& Wood (1968) in the $E-T$ plane is modified by rotation. We shall show that rotation advances the onset of gravothermal catastrophe in the microcanonical ensemble and delays the isothermal collapse in the canonical ensemble. Using the turning point criterion of Katz (1978), we argue that the series of equilibria becomes unstable at the point of minimum energy (in the microcanonical ensemble) or at the point of minimum temperature (in the canonical ensemble). Interestingly, these instabilities happen to be close, in each ensemble, to the point of maximum flattening. We generalize the singular isothermal solution to the case of a slowly rotating configuration. We also consider the case of slowly rotating self-gravitating fermions. This system exhibits phase transitions between "gaseous" states with an almost uniform distribution of matter and "condensed" states with a corehalo structure. By cooling below a critical temperature, an almost nonrotating gaseous sphere can collapse into a rotating "fermion ball" containing a large fraction of mass and angular momentum.

\section{Statistical mechanics of rotating self-gravitating systems}

\subsection{The mean-field approach}

Consider a system of $N$ particles, each of mass $m$, interacting via Newtonian gravity. The system is enclosed within a spherical box of radius $R$ which preserves the rotational symmetry of the original Hamiltonian. We allow the system to have a non vanishing angular momentum. Let $f(\boldsymbol{r}, \boldsymbol{v}, t)$ denote the distribution function of the system, i.e. $f(\boldsymbol{r}, \boldsymbol{v}, t) \mathrm{d}^{3} \boldsymbol{r} \mathrm{d}^{3} \boldsymbol{v}$ gives the mass of particles whose position and velocity are in the cell $\left(\boldsymbol{r}, \boldsymbol{v} ; \boldsymbol{r}+\mathrm{d}^{3} \boldsymbol{r}, \boldsymbol{v}+\mathrm{d}^{3} \boldsymbol{v}\right)$ at time $t$. The integral of $f$ over the velocity determines the spatial density

$\rho=\int f \mathrm{~d}^{3} \boldsymbol{v}$.

The mass and angular momentum of the configuration are given by

$M=\int \rho \mathrm{d}^{3} \boldsymbol{r}$

$\boldsymbol{L}=\int f \boldsymbol{r} \times \boldsymbol{v} \mathrm{d}^{3} \boldsymbol{r} \mathrm{d}^{3} \boldsymbol{v}$

On the other hand, in the mean-field approximation, the energy can be expressed as

$E=\frac{1}{2} \int f v^{2} \mathrm{~d}^{3} \boldsymbol{r} \mathrm{d}^{3} \boldsymbol{v}+\frac{1}{2} \int \rho \Phi \mathrm{d}^{3} \boldsymbol{r}=K+W$,

where $K$ is the kinetic energy and $W$ the potential energy. The gravitational potential $\Phi$ is related to the spatial density by the Newton-Poisson equation

$\Delta \Phi=4 \pi G \rho$.

The equilibrium configuration of the system is determined by maximizing the Boltzmann entropy

$S=-k \int \frac{f}{m} \ln \frac{f}{m} \mathrm{~d}^{3} \boldsymbol{r} \mathrm{d}^{3} \boldsymbol{v}$,

while conserving mass, angular momentum and energy. We stress that for systems interacting via a long-range potential, like gravity, the mean-field approximation is exact in a suitable thermodynamic limit such that $N \rightarrow \infty$ with $N / R$ fixed. Therefore, our variational procedure is entirely rigorous and provides a simple approach to the problem. We note, however, that the thermodynamic limit for self-gravitating systems is very unusual since the results still depend on the shape of the confining volume contrary to ordinary (extensive) systems. This peculiar feature has also been observed for other systems with long-range interactions like two-dimensional vortices in hydrodynamics (e.g., Chavanis \& Sommeria 1996).

Introducing Lagrange multipliers $\alpha, \beta$ and $-\beta \boldsymbol{\Omega}$ for each constraint, we find that the critical points of entropy are given by

$f=A^{\prime} \mathrm{e}^{-\beta\left(\frac{v^{2}}{2}+\Phi-\boldsymbol{\Omega} \cdot(\boldsymbol{r} \times \boldsymbol{v})\right)}$, 
where

$\beta=\frac{m}{k T}$.

We can rewrite the foregoing expression for $f$ in the more suggestive form

$f=A^{\prime} \mathrm{e}^{-\frac{1}{2} \beta(\boldsymbol{v}-\boldsymbol{\Omega} \times \boldsymbol{r})^{2}} \mathrm{e}^{-\beta\left(\Phi-\frac{1}{2}(\boldsymbol{\Omega} \times \boldsymbol{r})^{2}\right)}$.

We note that the most probable distribution of a rotating self-gravitating system is a Maxwell-Boltzmann law with a dispersion the same at every point (isothermal distribution). Moreover, the most probable form of rotation is a rigid rotation $\boldsymbol{\Omega}$. By integrating Eq. (9) over the velocity, we find that the density is given by the Boltzmann factor

$\rho=A \mathrm{e}^{-\beta\left(\Phi-\frac{1}{2}(\mathbf{\Omega} \times \boldsymbol{r})^{2}\right)}$.

The quantity in parenthesis is the "effective" potential in the rotating frame

$\Phi_{\text {eff }}=\Phi-\frac{1}{2}(\boldsymbol{\Omega} \times \boldsymbol{r})^{2}$,

accounting for inertial forces. The equilibrium configuration is obtained by solving the Boltzmann-Poisson system

$\Delta \Phi=4 \pi G A \mathrm{e}^{-\beta\left(\Phi-\frac{1}{2}(\mathbf{\Omega} \times \boldsymbol{r})^{2}\right)}$,

and relating the Lagrange multipliers $A, \beta$ and $\mathbf{\Omega}$ to the constraints $M, E$ and $\boldsymbol{L}$. The configuration is thermodynamically stable if the second order variations of entropy are negative for any perturbation that satisfies the constraints on mass, energy and angular momentum. This corresponds to the microcanonical description. Alternatively, we could describe the system in the canonical ensemble assuming that $\beta$ and $\boldsymbol{\Omega}$ are fixed, instead of $E$ and $\boldsymbol{L}$. In that case, the equilibrium configuration is obtained by maximizing the free energy $J=S-\beta E-\beta \boldsymbol{\Omega} \cdot \boldsymbol{L}$ at fixed $M, \beta$ and $\boldsymbol{\Omega}$. Of course, the microcanonical and canonical ensembles yield the same critical points, i.e. the critical points of entropy at fixed mass, energy and angular momentum and the critical points of free energy at fixed mass, temperature and angular velocity coincide. Only the onset of instability, regarding the second order variations of $S$ or $J$, will differ from one ensemble to the other. In the non-rotating case, the thermodynamical stability analysis was performed by Padmanabhan (1989) in the microcanonical ensemble and by Chavanis (2002a) in the canonical ensemble, by explicitly calculating the second order variations of the thermodynamical potential and reducing the stability problem to the study of an eigenvalue equation. The onset of instability can also be determined by the turning point criterion of Katz (1978) who extended the theory of Poincaré on linear series of equilibria.

\subsection{The rotating isothermal sphere}

To determine the structure of rotating isothermal spheres, we first introduce the function $\Psi=\beta\left(\Phi_{\text {eff }}-\Phi_{0}\right)$, where $\Phi_{0}$ is the gravitational potential at $r=0$. Then, the density field can be written

$\rho=\rho_{0} \mathrm{e}^{-\Psi}$, where $\rho_{0}$ is the central density. Introducing the notations $\xi=$ $\left(4 \pi G \beta \rho_{0}\right)^{1 / 2} r, v=\Omega^{2} / 2 \pi G \rho_{0}$ and using spherical coordinates $r, \theta, \phi$, we can rewrite the Boltzmann-Poisson Eq. (12) in the form

$\frac{1}{\xi^{2}} \frac{\partial}{\partial \xi}\left(\xi^{2} \frac{\partial \Psi}{\partial \xi}\right)+\frac{1}{\xi^{2}} \frac{\partial}{\partial \mu}\left(\left(1-\mu^{2}\right) \frac{\partial \Psi}{\partial \mu}\right)=\mathrm{e}^{-\Psi}-v$,

where $\mu=\cos \theta$ and we have neglected $\phi$ by assuming that the configuration is symmetric with respect to the axis $\boldsymbol{\Omega}=\Omega \boldsymbol{z}$. The true gravitational potential $\Phi$ is related to $\Psi$ by the relation

$\beta \Phi=\Psi+\frac{1}{4} v \xi^{2}\left(1-\mu^{2}\right)+\beta \Phi_{0}$

Equation (14) is the fundamental equation of the problem. For $v=0$ (no rotation), we recover the Emden equation

$\frac{1}{\xi^{2}} \frac{\mathrm{d}}{\mathrm{d} \xi}\left(\xi^{2} \frac{\mathrm{d} \psi}{\mathrm{d} \xi}\right)=\mathrm{e}^{-\psi}$,

with $\psi=\psi^{\prime}=0$ at $\xi=0$ (Chandrasekhar 1942).

So far, we have made no approximation regarding the value of the angular velocity. We shall now consider the case of slowly rotating structures and let $v \rightarrow 0$. Assuming the following form for our solution

$\Psi=\psi+v \Theta+v^{2} \Theta_{2}+\ldots$

and keeping terms only to first order in $v$, we find that $\Theta$ satisfies the equation

$\frac{1}{\xi^{2}} \frac{\partial}{\partial \xi}\left(\xi^{2} \frac{\partial \Theta}{\partial \xi}\right)+\frac{1}{\xi^{2}} \frac{\partial}{\partial \mu}\left(\left(1-\mu^{2}\right) \frac{\partial \Theta}{\partial \mu}\right)=-\Theta \mathrm{e}^{-\psi}-1$.

Now, following a procedure that dates back to Milne (1923) and Chandrasekhar (1933), we shall assume for $\Theta$ the following form

$\Theta=\phi_{0}(\xi)+\sum_{j=1}^{+\infty} A_{j} \phi_{j}(\xi) P_{j}(\mu)$,

where $P_{j}(\mu)$ are the Legendre polynomials satisfying the differential equation

$\frac{\partial}{\partial \mu}\left(\left(1-\mu^{2}\right) \frac{\partial P_{j}}{\partial \mu}\right)+j(j+1) P_{j}=0$.

Substituting for $\Theta$ from Eq. (19) in Eq. (18) and equating coefficients of $P_{j}$, we get

$\frac{1}{\xi^{2}} \frac{\mathrm{d}}{\mathrm{d} \xi}\left(\xi^{2} \frac{\mathrm{d} \phi_{0}}{\mathrm{~d} \xi}\right)=-\mathrm{e}^{-\psi} \phi_{0}-1$

$\frac{1}{\xi^{2}} \frac{\mathrm{d}}{\mathrm{d} \xi}\left(\xi^{2} \frac{\mathrm{d} \phi_{j}}{\mathrm{~d} \xi}\right)=\left(\frac{j(j+1)}{\xi^{2}}-\mathrm{e}^{-\psi}\right) \phi_{j}, \quad(j=1,2, \ldots)$

with $\phi_{j}=\phi_{j}^{\prime}=0$ at $\xi=0$.

So far, the $A_{j}$ are arbitrary. They will be determined by requiring that the gravitational potential and its radial derivative are continuous across the sphere at $r=R$. Now, outside the sphere the potential is given by the Laplace equation

$\Delta \Phi_{\mathrm{ext}}=0$. 
The physically acceptable solution of this equation tending to zero at infinity can be written as

$\beta \Phi_{\mathrm{ext}}=\frac{B_{0}}{\xi}+v \sum_{j=1}^{+\infty} \frac{B_{j}}{\xi^{j+1}} P_{j}(\mu)$.

On the other hand, according to Eq. (15), we have inside the spherical box

$\beta \Phi_{\text {int }}=\Psi+\frac{1}{6} v \xi^{2}\left(1-P_{2}(\mu)\right)+\beta \Phi_{0}$,

where $\Psi(\xi, \mu)$ is given by Eqs. (17) and (19) and use has been made of the identity $1-\mu^{2}=\frac{2}{3}\left(1-P_{2}(\mu)\right)$. Let us denote by

$\alpha=\left(4 \pi G \beta \rho_{0}\right)^{1 / 2} R$,

the value of $\xi$ at $r=R$. Comparing the inner and the external potentials at $\xi=\alpha$, and also their derivative, we find that $A_{j}=$ $B_{j}=0$ if $j \neq 2$ and, for $j=2$,

$\frac{B_{2}}{\alpha^{3}}=A_{2} \phi_{2}(\alpha)-\frac{1}{6} \alpha^{2}$,

$-\frac{3 B_{2}}{\alpha^{4}}=A_{2} \phi_{2}^{\prime}(\alpha)-\frac{1}{3} \alpha$.

Solving for $A_{2}$, we get

$A_{2}=\frac{5}{6} \frac{\alpha^{2}}{3 \phi_{2}(\alpha)+\alpha \phi_{2}^{\prime}(\alpha)}$.

Therefore, the solution of the Boltzmann-Poisson Eq. (14) to first order in $v$ is given by

$\Psi=\psi(\xi)+v\left\{\phi_{0}(\xi)+\frac{5}{6} \frac{\alpha^{2}}{3 \phi_{2}(\alpha)+\alpha \phi_{2}^{\prime}(\alpha)} \phi_{2}(\xi) P_{2}(\mu)\right\}$

with

$\frac{1}{\xi^{2}} \frac{\mathrm{d}}{\mathrm{d} \xi}\left(\xi^{2} \frac{\mathrm{d} \phi_{0}}{\mathrm{~d} \xi}\right)=-\mathrm{e}^{-\psi} \phi_{0}-1$,

$\frac{1}{\xi^{2}} \frac{\mathrm{d}}{\mathrm{d} \xi}\left(\xi^{2} \frac{\mathrm{d} \phi_{2}}{\mathrm{~d} \xi}\right)=\left(\frac{6}{\xi^{2}}-\mathrm{e}^{-\psi}\right) \phi_{2}$

with $\phi_{j}=\phi_{j}^{\prime}=0$ at $\xi=0$.

\subsection{The slowly rotating singular isothermal sphere}

For $\xi \rightarrow+\infty$, the solution of the Emden Eq. (16) behaves like (Chandrasekhar 1942)

$\mathrm{e}^{-\psi} \sim \frac{2}{\xi^{2}}$

Substituting this asymptotic behaviour in Eq. (31) and making the change of variables $\xi=\mathrm{e}^{t}$, we obtain

$\frac{\mathrm{d}^{2} \phi_{0}}{\mathrm{~d} t^{2}}+\frac{\mathrm{d} \phi_{0}}{\mathrm{~d} t}+2 \phi_{0}=-\mathrm{e}^{2 t}$

The solution of this equation is readily found. After returning to original variables, we get for large $\xi$ 's:

$\phi_{0}=-\frac{1}{8} \xi^{2}+\frac{A}{\xi^{1 / 2}} \cos \left(\frac{\sqrt{7}}{2} \ln \xi+\delta\right)$.
Following the same procedure for the function $\phi_{2}$, we have

$\frac{\mathrm{d}^{2} \phi_{2}}{\mathrm{~d} t^{2}}+\frac{\mathrm{d} \phi_{2}}{\mathrm{~d} t}-4 \phi_{2}=0$

leading to

$\phi_{2}=\frac{A^{\prime}}{\xi^{1 / 2}} \cosh \left(\frac{\sqrt{17}}{2} \ln \xi+\delta^{\prime}\right)$.

Keeping only the leading term in Eqs. (35) and (37) in the limit $\xi \rightarrow+\infty$, we find that

$\phi_{0} \sim-\frac{1}{8} \xi^{2} ; \quad \phi_{2} \sim K \xi^{\gamma}, \quad \gamma=\frac{\sqrt{17}}{2}-\frac{1}{2}$.

Substituting these results in Eq. (30) and returning to dimensional variables, we finally obtain

$\rho=\frac{1}{2 \pi G \beta r^{2}}+\frac{\Omega^{2}}{8 \pi G}\left\{1-\frac{20}{3(3+\gamma)}\left(\frac{R}{r}\right)^{2-\gamma} P_{2}(\mu)\right\}$,

which is the expression of the slowly rotating singular isothermal sphere.

\section{The thermodynamical parameters}

\subsection{The mass}

In spherical coordinates, the mass is given by

$M=2 \pi \int_{-1}^{+1} \int_{0}^{R} \rho r^{2} \mathrm{~d} r \mathrm{~d} \mu$.

Introducing the dimensionless variables previously defined, we obtain, using Eq. (13),

$\eta \equiv \frac{\beta G M}{R}=\frac{1}{2 \alpha} \int_{-1}^{+1} \int_{0}^{\alpha} \mathrm{e}^{-\Psi} \xi^{2} \mathrm{~d} \xi \mathrm{d} \mu$.

Substituting for $\Psi$ from Eq. (30) in Eq. (41) and recalling that $\int_{-1}^{+1} P_{j}(\mu) \mathrm{d} \mu=0$, we obtain to first order in $v$ :

$\eta=\frac{1}{\alpha} \int_{0}^{\alpha} \mathrm{e}^{-\psi}\left(1-v \phi_{0}(\xi)\right) \xi^{2} \mathrm{~d} \xi$

Now,

$\int_{0}^{\alpha} \mathrm{e}^{-\psi} \xi^{2} \mathrm{~d} \xi=\int_{0}^{\alpha} \mathrm{d} \xi \frac{\mathrm{d}}{\mathrm{d} \xi}\left(\xi^{2} \frac{\mathrm{d} \psi}{\mathrm{d} \xi}\right)=\alpha^{2} \psi^{\prime}(\alpha)$,

$$
\begin{array}{r}
\int_{0}^{\alpha} \mathrm{e}^{-\psi} \phi_{0} \xi^{2} \mathrm{~d} \xi=-\int_{0}^{\alpha} \mathrm{d} \xi \\
\left\{\xi^{2}+\frac{\mathrm{d}}{\mathrm{d} \xi}\left(\xi^{2} \frac{\mathrm{d} \phi_{0}}{\mathrm{~d} \xi}\right)\right\} \\
=-\frac{\alpha^{3}}{3}-\alpha^{2} \phi_{0}^{\prime}(\alpha) .
\end{array}
$$

Therefore, the normalized inverse temperature $\eta$ is expressed in terms of $\alpha$ and $v$ by the relation

$\eta=\alpha \psi^{\prime}(\alpha)+v\left[\frac{\alpha^{2}}{3}+\alpha \phi_{0}^{\prime}(\alpha)\right]$

Expressing the central density in terms of $\alpha$, using Eq. (26), we find that

$v=\frac{2 \eta}{\alpha^{2}} \omega^{2}, \quad$ with $\quad \omega=\Omega\left(\frac{R^{3}}{G M}\right)^{1 / 2}$. 
Therefore, the foregoing expression for $\eta$ can be rewritten to order $\omega^{2}$ :

$\eta=\alpha \psi^{\prime}(\alpha)\left\{1+2 \omega^{2}\left[\frac{1}{3}+\frac{1}{\alpha} \phi_{0}^{\prime}(\alpha)\right]\right\}$.

The validity of our perturbative approach requires that

$\epsilon \equiv 2 \omega^{2}\left[\frac{1}{3}+\frac{1}{\alpha} \phi_{0}^{\prime}(\alpha)\right] \ll 1$.

\subsection{The angular momentum}

Using the Maxwell-Boltzmann distribution (9), we can rewrite the angular momentum (3) in the form

$\boldsymbol{L}=\int \rho \boldsymbol{r} \times \boldsymbol{u} \mathrm{d}^{3} \boldsymbol{r}, \quad$ with $\quad \boldsymbol{u}=\boldsymbol{\Omega} \times \boldsymbol{r}$.

After straightforward manipulations, we find that the angular momentum is related to the angular velocity by

$L_{i}=I_{i j} \Omega_{j}$

where $I_{i j}=\int \rho\left(r^{2} \delta_{i j}-r_{i} r_{j}\right) \mathrm{d}^{3} \boldsymbol{r}$ is the moment of inertia tensor. If the density depends only on $r$ and $\theta$, we have $\boldsymbol{L}=I \mathbf{\Omega}$ with

$I=2 \pi \int_{-1}^{+1} \int_{0}^{R} \rho r^{4}\left(1-\mu^{2}\right) \mathrm{d} r \mathrm{~d} \mu$.

For our purposes, it is sufficient to determine the angular momentum to first order in $\Omega$. Therefore, we need just the expression of the moment of inertia $I$ for a non-rotating isothermal sphere. Introducing the dimensionless variables previously defined, and using the expression (47) for $\eta$, we get

$\mathcal{I} \equiv \frac{I}{M R^{2}}=\frac{2}{3 \alpha^{4} \psi^{\prime}(\alpha)} \int_{0}^{\alpha} \mathrm{e}^{-\psi} \xi^{4} \mathrm{~d} \xi$

Then,

$\lambda=\mathcal{I}(\alpha) \omega, \quad$ with $\quad \lambda=\frac{L}{\sqrt{G M^{3} R}}$.

\subsection{The energy}

Quite generally, the potential energy of a self-gravitating system can be written in the form (see, e.g., Binney \& Tremaine 1987)

$W=-\int \rho \boldsymbol{r} \cdot \nabla \Phi \mathrm{d}^{3} \boldsymbol{r}$.

It can be readily verified that the condition $f=f\left(\frac{w^{2}}{2}+\Phi_{\text {eff }}\right)$, where $\boldsymbol{w}=\boldsymbol{v}-\boldsymbol{\Omega} \times \boldsymbol{r}$ and $\Phi_{\mathrm{eff}}$ is the effective potential (11), is equivalent to the condition of hydrostatic equilibrium in the rotating frame

$\nabla p=-\rho \nabla \Phi-\rho \mathbf{\Omega} \times(\boldsymbol{\Omega} \times \boldsymbol{r})$,

where

$p=\frac{1}{3} \int f w^{2} \mathrm{~d}^{3} w$ is the local pressure. For an isothermal gas $p=\frac{k}{m} \rho T$. Inserting Eq. (55) in Eq. (54), we obtain after straightforward calculations

$W+3 \int p \mathrm{~d}^{3} \boldsymbol{r}+\boldsymbol{L} \cdot \boldsymbol{\Omega}-\oint p \boldsymbol{r} \cdot \mathrm{d} \boldsymbol{S}=0$,

where $\mathrm{d} S$ is a surface element normal to the spherical box. On the other hand, we can write the kinetic energy in the form

$K=K_{\text {rot }}+K_{\text {th }}$

where

$K_{\mathrm{rot}}=\frac{1}{2} \int \rho u^{2} \mathrm{~d}^{3} \boldsymbol{r}=\frac{1}{2} \boldsymbol{L} \cdot \boldsymbol{\Omega}$,

is the rotational energy and

$K_{\mathrm{th}}=\frac{1}{2} \int f w^{2} \mathrm{~d}^{3} \boldsymbol{r} \mathrm{d}^{3} w=\frac{3}{2} \int p \mathrm{~d}^{3} \boldsymbol{r}$,

is the thermal energy. For an isothermal gas $K_{\mathrm{th}}=\frac{3}{2} N k T$. Combining the foregoing relations, Eq. (57) becomes

$W+2 K=\oint p \boldsymbol{r} \cdot \mathrm{d} S$

which is the Virial theorem for a self-gravitating gas enclosed within a box. For the total energy $E=K+W$, we have

$E=-K+\oint p \boldsymbol{r} \cdot \mathrm{d} \boldsymbol{S}$.

This expression is completely general. If, now, the density depends only on $r$ and $\theta$, and if the gas is isothermal, it is possible to combine Eq. (62) with the formulae derived in Sect. 2.2 to obtain the expression

$\Lambda \equiv-\frac{E R}{G M^{2}}=\frac{3}{2 \eta}+\frac{1}{2} \lambda \omega-\frac{\alpha^{2}}{\eta^{2}} \mathrm{e}^{-\psi(\alpha)}\left(1-v \phi_{0}(\alpha)\right)$,

where $\eta$ and $v$ can be expressed in terms of $\alpha$ and $\omega$ by Eqs. (46) and (47). We also recall that our theory is valid to order $\omega^{2}$. For $\omega=0$, Eqs. (47) and (63) reduce to the equations of state obtained by Lynden-Bell \& Wood (1968) for non-rotating isothermal spheres.

\subsection{The entropy}

Using Eqs. (9), (10) and (13), the distribution function of a rotating isothermal gas can be written

$f=\left(\frac{\beta}{2 \pi}\right)^{3 / 2} \mathrm{e}^{-\beta \frac{w^{2}}{2}} \rho_{0} \mathrm{e}^{-\Psi}$.

Substituting this expression in Eq. (6) and expressing $\rho_{0}$ in terms of $\alpha$, we get

$\frac{m S}{k}=-\frac{1}{2} M \ln \beta-2 M \ln \alpha+\int \rho \Psi \mathrm{d}^{3} \boldsymbol{r}$.

Throughout the paper, we shall not write the constant terms (depending on the fixed parameters $M$ and $R$ ) entering in the expression of the entropy. Therefore, a term $\beta K_{\mathrm{th}}=\frac{3}{2} M$ has 
been ignored in Eq. (65). Using the definition of $\Psi$, the last integral can be rewritten

$\int \rho \Psi \mathrm{d}^{3} \boldsymbol{r}=2 \beta W-\beta K_{\mathrm{rot}}-\beta M \Phi_{0}$.

Using $W=E-K_{\text {rot }}-K_{\text {th }}$, we obtain

$\frac{m S}{k}=-\frac{1}{2} M \ln \beta-\frac{3}{2} \beta \boldsymbol{L} \cdot \boldsymbol{\Omega}-2 M \ln \alpha+2 \beta E-\beta M \Phi_{0}$.

Introducing the dimensionless parameters previously defined, we get

$\frac{S}{N k}=-\frac{1}{2} \ln \eta-\frac{3}{2} \eta \lambda \omega-2 \ln \alpha-2 \eta \Lambda-\beta \Phi_{0}$.

We now need to determine the central potential $\Phi_{0}$. The condition that $\Phi$ and $\partial \Phi / \partial \xi$ are continuous at $\xi=\alpha$ implies for $j=0$ :

$\frac{B_{0}}{\alpha}=\psi(\alpha)+v \phi_{0}(\alpha)+\frac{1}{6} v \alpha^{2}+\beta \Phi_{0}$,

$-\frac{B_{0}}{\alpha^{2}}=\psi^{\prime}(\alpha)+v \phi_{0}^{\prime}(\alpha)+\frac{1}{3} v \alpha$.

Comparing Eq. (70) with Eq. (45), we see that $-B_{0} / \alpha=\eta$. Inserting this result in Eq. (69), we obtain

$-\beta \Phi_{0}=\eta+\psi(\alpha)+v\left[\frac{\alpha^{2}}{6}+\phi_{0}(\alpha)\right]$.

With this new relation, the entropy (68) becomes

$$
\begin{array}{r}
\frac{S}{N k}=-\frac{1}{2} \ln \eta-2 \ln \alpha+\psi(\alpha)+\eta-2 \Lambda \eta \\
-\frac{3}{2} \eta \lambda \omega+v\left[\frac{\alpha^{2}}{6}+\phi_{0}(\alpha)\right] .
\end{array}
$$

\subsection{The flattening function}

The value of the potential at $\xi$ for a non-rotating configuration is $\psi(\xi)$. For a rotating configuration, the equation of the surface with the same value of the potential is given, to first order in $v$, by

$\xi^{\prime}=\xi-\frac{v}{\psi^{\prime}(\xi)}\left\{\phi_{0}(\xi)+\frac{5}{6} \frac{\alpha^{2}}{3 \phi_{2}(\alpha)+\alpha \phi_{2}^{\prime}(\alpha)} \phi_{2}(\xi) P_{2}(\mu)\right\}$

If $a$ denotes the largest radius of the isodensity surface (at the equator $\mu=0$ ) and $b$ the smallest radius (at the pole $\mu=1$ ), and if we define the flattening by $f=1-b / a$ (see, e.g., Lagoute \& Longaretti 1996), we get

$f(\xi)=\frac{5}{4} \frac{v}{\xi \psi^{\prime}(\xi)} \frac{\alpha^{2}}{3 \phi_{2}(\alpha)+\alpha \phi_{2}^{\prime}(\alpha)} \phi_{2}(\xi)$.

Considering the limit $\xi \rightarrow+\infty$ and returning to dimensional variables, we find that the flattening function of the rotating singular isothermal sphere is

$f(r)=\frac{5}{4} \frac{\beta \Omega^{2} R^{2}}{3+\gamma}\left(\frac{r}{R}\right)^{\gamma}$,

a result which can also be derived directly from Eq. (39).
Coming back to Eq. (74), we find that the flattening behaves with the distance (for a given value of $\alpha$ ) as

$F(\xi)=-\frac{\phi_{2}(\xi)}{\xi \psi^{\prime}(\xi)}$

On the other hand, the flattening at the edge of the configuration $(\xi=\alpha)$ is given by

$f(\alpha)=\frac{5}{2} \omega^{2} \frac{\phi_{2}(\alpha)}{3 \phi_{2}(\alpha)+\alpha \phi_{2}^{\prime}(\alpha)}$,

where we have used Eqs. (46) and (47) to eliminate the variable $v$ in profit of $\omega$. In the canonical ensemble (fixed $\omega$ ), this function depends on $\alpha$ as

$F_{\mathrm{CE}}(\alpha)=\frac{\phi_{2}(\alpha)}{3 \phi_{2}(\alpha)+\alpha \phi_{2}^{\prime}(\alpha)}$

In the microcanonical ensemble (fixed $\lambda$ ), we must express the angular velocity in terms of the angular momentum using the relation (53). In that case, the flattening at the edge of the configuration depends on $\alpha$ as

$F_{\mathrm{MCE}}(\alpha)=\frac{1}{\mathcal{I}(\alpha)^{2}} F_{\mathrm{CE}}(\alpha)$.

We shall come back to these results in the following section.

\section{Equilibrium phase diagram}

\subsection{Microcanonical ensemble}

The microcanonical ensemble corresponds to isolated systems characterized by their energy $\Lambda$ and their angular momentum $\lambda$. In order to determine the caloric curve $\eta(\Lambda)$ for different values of $\lambda$, we need to solve Eqs. (16), (31) and (32) numerically. Expanding the functions $\psi, \phi_{0}$ and $\phi_{2}$ in Taylor series for $\xi \rightarrow$ 0 , we get

$\psi=\frac{1}{6} \xi^{2}-\frac{1}{120} \xi^{4}+\ldots$

$\phi_{0}=-\frac{1}{6} \xi^{2}+\frac{1}{120} \xi^{4}+\ldots$

$\phi_{2}=-\xi^{2}+\frac{1}{14} \xi^{4}+\ldots$

so that $\psi^{\prime \prime}(0)=1 / 3, \phi_{0}^{\prime \prime}(0)=-1 / 3$ and $\phi_{2}^{\prime \prime}(0)=-2$. The integration can be continued numerically by a standard RungeKutta routine. In Figs. 1-3, we plot the curves $\mathcal{I}(\alpha), \Lambda(\alpha)$ and $\eta(\alpha)$ defined by Eqs. (52), (63) and (47). These curves exhibit damped oscillations and tend to the values

$\mathcal{I}_{\mathrm{s}}=\frac{2}{9}, \quad \eta_{\mathrm{s}}=2+\frac{27}{4} \lambda^{2}, \quad \Lambda_{\mathrm{s}}=\frac{1}{4}-\frac{63}{32} \lambda^{2}$,

as $\alpha \rightarrow+\infty$. These asymptotic limits correspond to the singular solution (39). The iso-density contours of the rotating singular isothermal sphere are represented in Fig. 4 (for $\lambda=0.17$ ).

In Fig. 5, we have represented the caloric curve $\eta(\Lambda)$. It has a classical spiral behaviour as noted by a number of authors in the non-rotating case. There is no equilibrium state (i.e., no critical point of entropy) above the value $\Lambda_{c}(\lambda)$. In that case, 


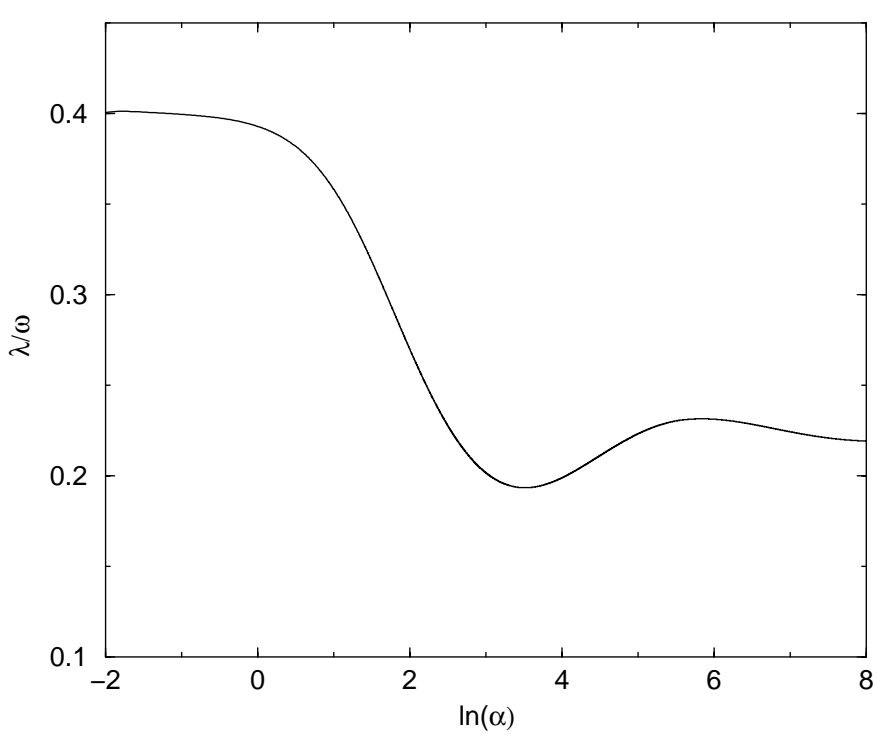

Fig. 1. Moment of inertia for a non-rotating isothermal sphere along the series of equilibria (parametrized by $\alpha$ ).

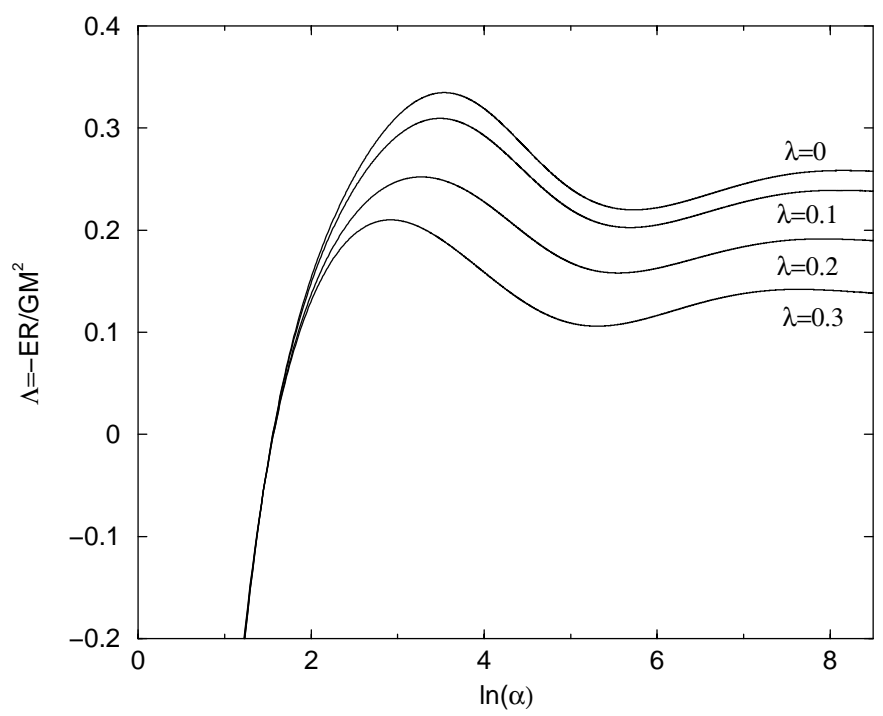

Fig. 2. Normalized energy of an isothermal sphere along the series of equilibria (parametrized by $\alpha$ ) for different values of the angular momentum $\lambda=0,0.1,0.2,0.3$.

the system will collapse and overheat (gravothermal catastrophe). It is also at this point that the series of equilibria becomes unstable (saddle points of entropy) in the microcanonical ensemble (Katz 1978). We see that rotation tends to favour the instability, i.e., the gravothermal catastrophe occurs sooner than in the non-rotating case (see also Fig. 2).

In Fig. 6, we have represented the angular velocity of the system as a function of energy for different values of the angular momentum. We observe that the curve has a spiral behaviour similar to the $\eta-\Lambda$ diagram, but reversed. We might expect that the moment of inertia decreases as the system becomes more and more concentrated, resulting in an increase of angular velocity along the series of equilibria. This is true for moderate density contrasts (up to $\sim 709$ ), coinciding with the region of stability, but not for larger density contrasts. Indeed,

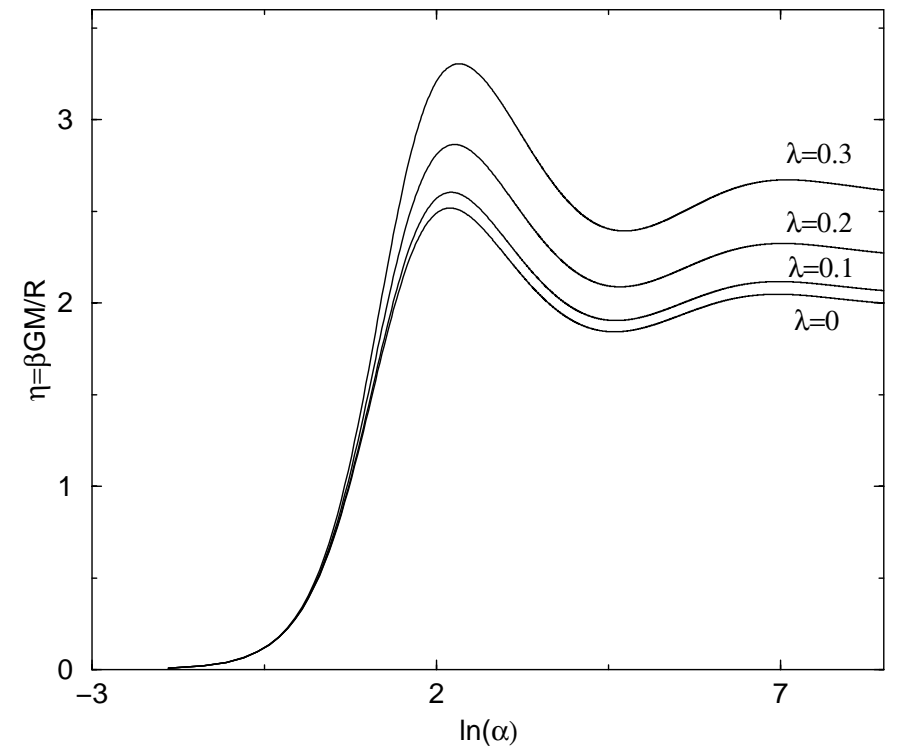

Fig. 3. Normalized inverse temperature of an isothermal sphere along the series of equilibria (parametrized by $\alpha$ ) for different values of the angular momentum $\lambda=0,0.1,0.2,0.3$.

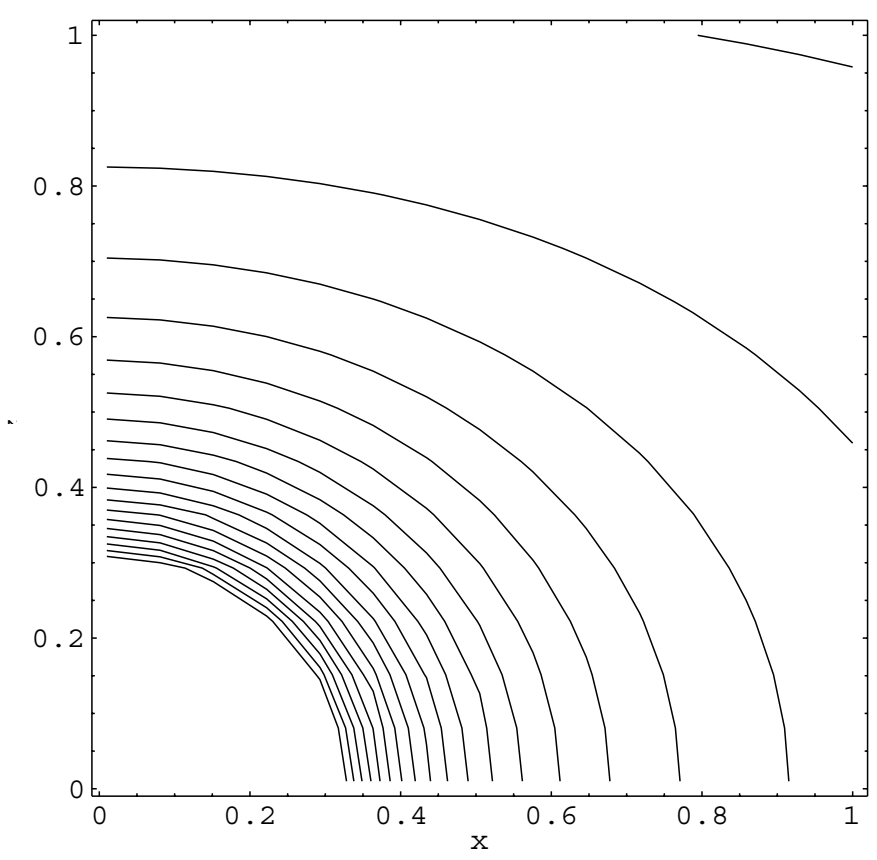

Fig. 4. Iso-density contours of the rotating singular isothermal sphere with an angular momentum $\lambda=0.17$.

although the central density tends to diverge, the mass contained in the core is low and does not dominate the moment of inertia. Therefore, the moment of inertia and the angular velocity have a non monotonic (in fact oscillatory) behaviour with $\alpha$ and saturate to finite values $\mathcal{I}_{\mathrm{s}}$ and $\omega_{\mathrm{s}}=\lambda / \mathcal{I}_{\mathrm{s}}$ as $\alpha \rightarrow+\infty$.

In Fig. 7, we plot the flattening function $F(\xi)$ defined by Eq. (76). As expected, the flattening is a monotonic function of the distance. For $\xi \rightarrow 0, F \rightarrow 3$ and for $\xi \rightarrow+\infty, F \sim \xi^{\gamma}$. In Fig. 8, we plot the flattening at the edge of the configuration $F_{\mathrm{MCE}}(\alpha)$ as a function of $\alpha$ in the microcanonical ensemble (see Eq. (79)). We observe that the curve displays damped 


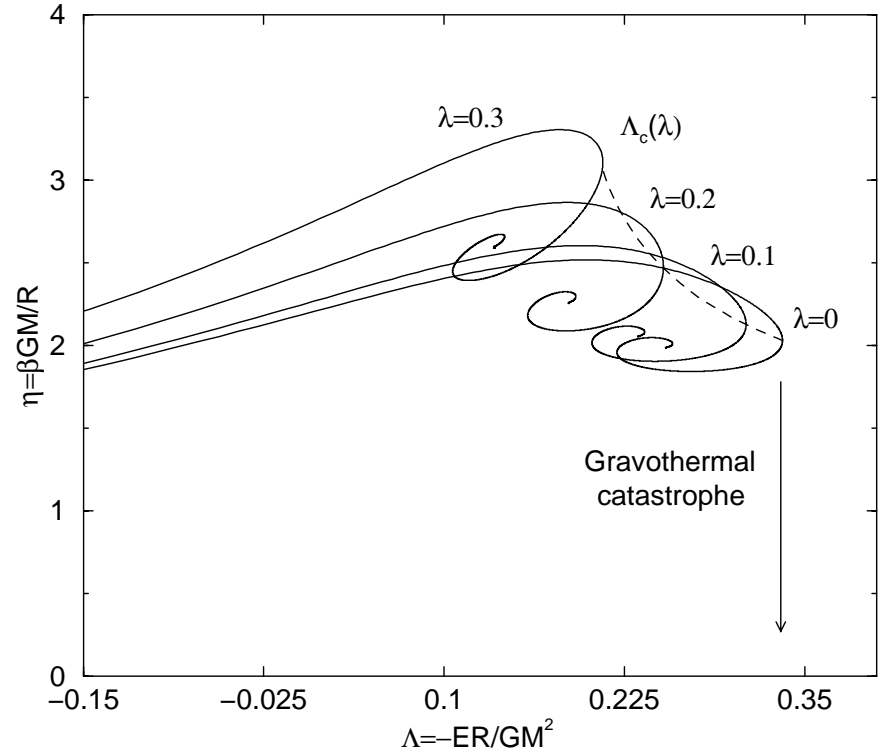

Fig. 5. Caloric curve of rotating spheres giving the inverse temperature $\eta$ as a function of minus the energy $\Lambda$ for different values of the angular momentum $\lambda$. The gravothermal instability at $\Lambda_{c}$ occurs sonner (i.e. for larger energies) when the system is rotating.

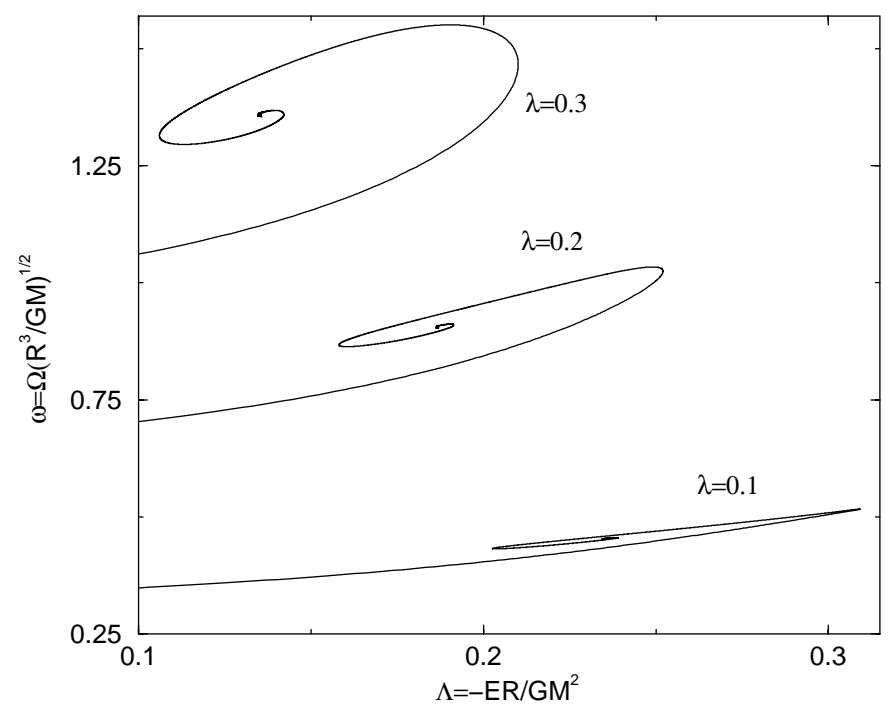

Fig. 6. Angular velocity $\omega$ of the system as a function of energy $\Lambda$ for different values of the angular momentum $\lambda$.

oscillations towards the asymptotic value $\frac{1}{3+\gamma}(9 / 2)^{2}$. In particular, the flattening (by unit of $\lambda^{2}$ ) is maximum for $\alpha=32.6 \ldots$ Interestingly, this value lies precisely in the range of values at which the gravothermal catastrophe sets in (compare Fig. 8 with Fig. 2 giving the energy along the series of equilibria).

\subsection{Canonical ensemble}

The canonical ensemble is characterized by the specification of the inverse temperature $\eta$ and the angular velocity $\omega$. In Fig. 9, we have represented the curve $\Lambda(\eta)$ for different values of $\omega$. There is no equilibrium state (i.e., no critical point of free energy) above the value $\eta_{\mathrm{c}}(\omega)$. In that case, the system will undergo an isothermal collapse. It is also at this point that the

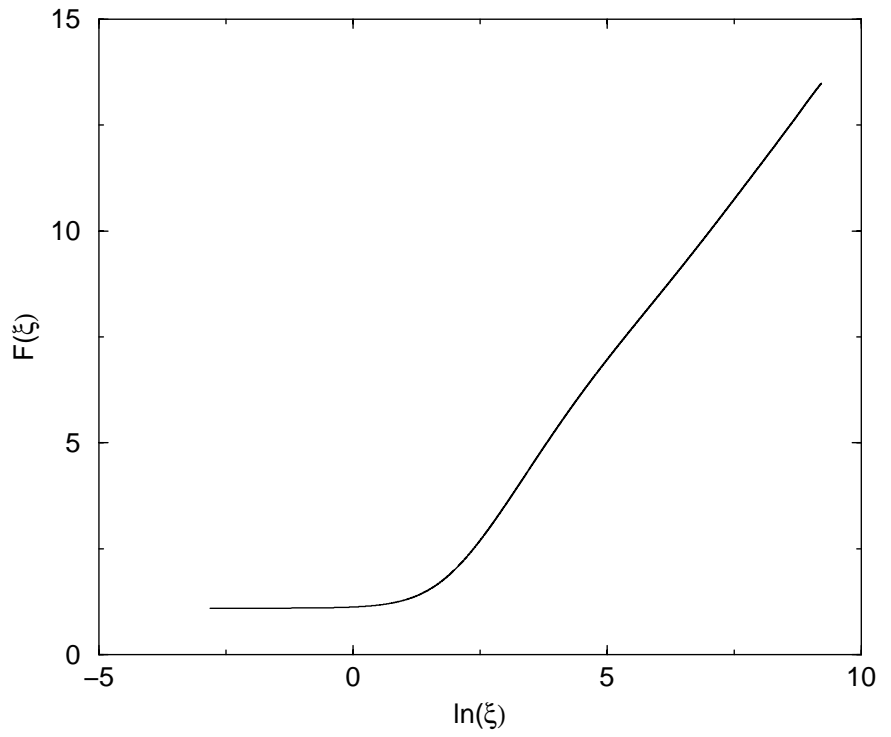

Fig. 7. Spatial dependance of the flattening function $F(\xi)$.

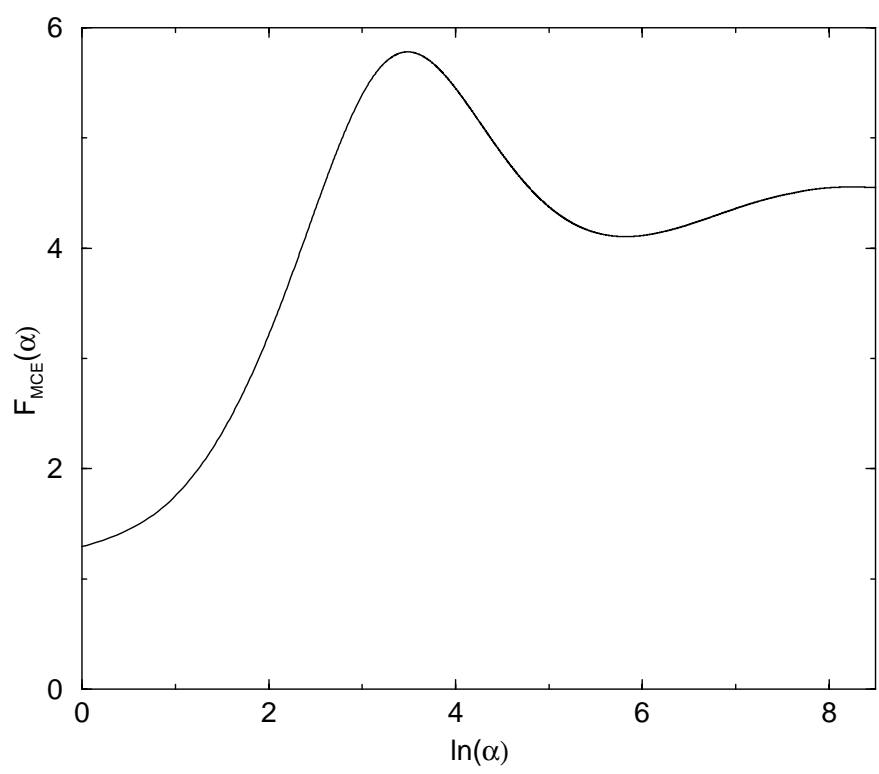

Fig. 8. Flattening at the edge of the configuration (per unit angular momentum squared) along the series of equilibria in the microcanonical ensemble. The flattening is maximum for $\alpha=32.6$. This value lies typically in the region where the gravothermal catastrophe sets in.

series of equilibria becomes unstable (saddle points of free energy) in the canonical ensemble (Katz 1978). We see that rotation tends to delay the instability, i.e., the isothermal collapse occurs later than in the non-rotating case.

In Fig. 10, we plot the flattening at the edge of the configuration $F_{\mathrm{CE}}(\alpha)$ as a function of $\alpha$ in the canonical ensemble (see Eq. (78)). The curve displays damped oscillations towards the value $\frac{1}{3+\gamma}$. The flattening (by unit of $\omega^{2}$ ) is maximum for $\alpha=5.4 \ldots$ Interestingly, this value is close to the typical values at which the isothermal collapse sets in (compare Fig. 10 with Fig. 11 giving the inverse temperature along the series of equilibria). 


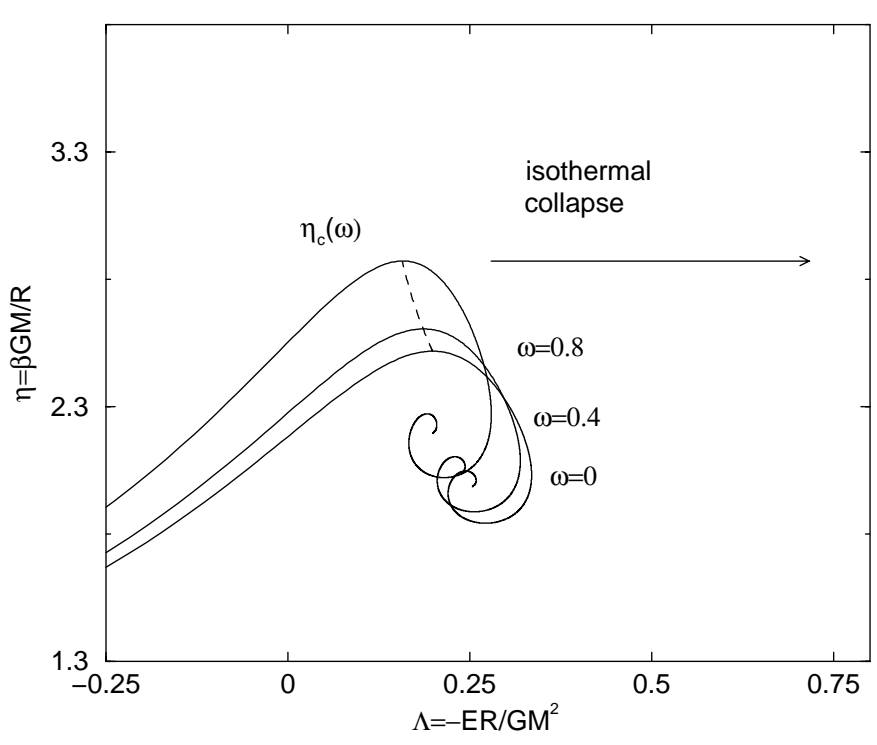

Fig. 9. Caloric curve of rotating spheres giving minus the energy $\Lambda$ as a function of the inverse temperature $\eta$ for different values of angular velocity $\omega$. The isothermal collapse at $\eta_{\mathrm{c}}(\omega)$ occurs later (i.e. for smaller temperatures) when the system is rotating.

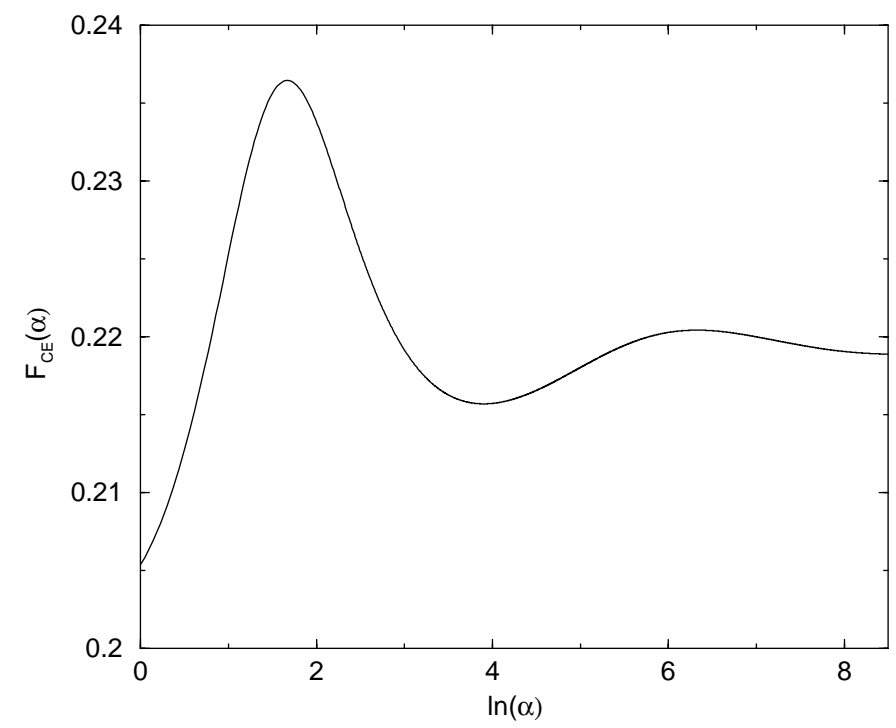

Fig. 10. Flattening at the edge of the configuration (per unit of angular velocity squared) along the series of equilibria in the canonical ensemble. The flattening is maximum for $\alpha=5.4$. This value lies typically in the region where the isothermal collapse sets in.

\section{Rotating self-gravitating fermions}

The previous results can be easily generalized to the case of self-gravitating fermions. This extension is relatively straightforward and we shall just give the main steps of the calculations. The thermodynamical parameters of a non-rotating Fermi gas at finite temperature have been calculated by Chavanis \& Sommeria (1998) and we shall adopt a similar presentation. For a rotating configuration, the Fermi-Dirac distribution can be written

$f=\frac{\eta_{0}}{1+\lambda \mathrm{e}^{\beta \Phi_{\text {eff }}} \mathrm{e}^{\beta \frac{w^{2}}{2}}}$,

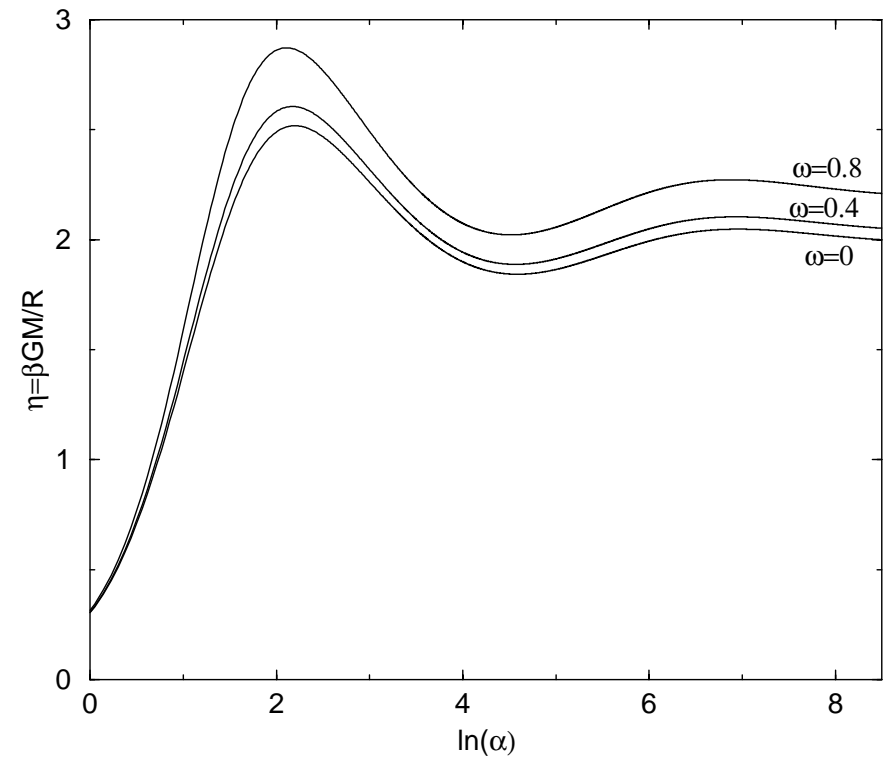

Fig. 11. Normalized inverse temperature of an isothermal sphere along the series of equilibria (parametrized by $\alpha$ ) for different values of angular velocity $\omega=0,0.4,0.8$. The series of equilibria becomes unstable after the first maximum.

where $\eta_{0}$ is the maximum allowable value of the distribution function and $\lambda>0$ a strictly positive parameter (inverse of the fugacity) insuring that $f \leq \eta_{0}$ (the other quantities have been defined previously). For quantum particles with spin $s$, $\eta_{0}=(2 s+1) m^{4} /(2 \pi \hbar)^{3}$. The distribution function (84) can be obtained by maximizing the Fermi-Dirac entropy

$S=-\int\left\{\frac{f}{\eta_{0}} \ln \frac{f}{\eta_{0}}+\left(1-\frac{f}{\eta_{0}}\right) \ln \left(1-\frac{f}{\eta_{0}}\right)\right\} \mathrm{d}^{3} \boldsymbol{r} \mathrm{d}^{3} \boldsymbol{v}$

at fixed mass, energy and angular momentum. This form of entropy also occurs in the context of violent relaxation for collisionless self-gravitating systems (Lynden-Bell 1967; Chavanis et al. 1996; Chavanis \& Sommeria 1998). Defining $\Psi=$ $\beta\left(\Phi_{\text {eff }}-\Phi_{0}\right)$ and $k=\lambda \mathrm{e}^{\beta \Phi_{0}}$, the spatial density can be written

$\rho=\rho_{0} \frac{I_{1 / 2}\left(k \mathrm{e}^{\Psi}\right)}{I_{1 / 2}(k)}, \quad$ with $\quad \rho_{0}=\frac{4 \pi \sqrt{2} \eta_{0}}{\beta^{3 / 2}} I_{1 / 2}(k)$,

where $I_{1 / 2}$ is the Fermi integral

$I_{n}(t)=\int_{0}^{+\infty} \frac{x^{n}}{1+t \mathrm{e}^{x}} \mathrm{~d} x$

of order $n=1 / 2$. Substituting the relation (86) in the Poisson Eq. (5) and introducing the dimensionless parameters $\xi=$ $\left(4 \pi G \beta \rho_{0}\right)^{1 / 2} r$ and $v=\frac{\Omega^{2}}{2 \pi G \rho_{0}}$, we obtain

$\frac{1}{\xi^{2}} \frac{\partial}{\partial \xi}\left(\xi^{2} \frac{\partial \Psi}{\partial \xi}\right)+\frac{1}{\xi^{2}} \frac{\partial}{\partial \mu}\left(\left(1-\mu^{2}\right) \frac{\partial \Psi}{\partial \mu}\right)=\frac{I_{1 / 2}\left(k \mathrm{e}^{\Psi}\right)}{I_{1 / 2}(k)}-v$

The relation between $\Psi$ and the true gravitational potential is still given by Eq. (15). For $v=0$, Eq. (88) reduces to (Chavanis \& Sommeria 1998):

$\frac{1}{\xi^{2}} \frac{\mathrm{d}}{\mathrm{d} \xi}\left(\xi^{2} \frac{\mathrm{d} \psi}{\mathrm{d} \xi}\right)=\frac{I_{1 / 2}\left(k \mathrm{e}^{\psi}\right)}{I_{1 / 2}(k)}$ 
with $\psi=\psi^{\prime}=0$ at $\xi=0$. For $k \rightarrow+\infty$ (classical limit), we can use the limiting form of the Fermi integral

$I_{n}(t) \sim \frac{1}{t} \Gamma(n+1), \quad(t \rightarrow+\infty)$

and we recover the classical Emden Eq. (16). For $k \rightarrow 0$ (completely degenerate limit), we have

$I_{n}(t) \sim \frac{(-\ln t)^{n+1}}{n+1}, \quad(t \rightarrow 0)$

and the differential Eq. (89) becomes equivalent to the LaneEmden equation for a polytrope of index $3 / 2$.

We shall now consider the case of slowly rotating structures and let $v \rightarrow 0$. Repeating the steps of Sect. 2.2, we find that $\psi(\xi, \mu)$ is given by Eq. (30) with the new functions $\phi_{0}$ and $\phi_{2}$ defined by

$\frac{1}{\xi^{2}} \frac{\mathrm{d}}{\mathrm{d} \xi}\left(\xi^{2} \frac{\mathrm{d} \phi_{0}}{\mathrm{~d} \xi}\right)=-\frac{1}{2} \frac{I_{-1 / 2}\left(k \mathrm{e}^{\psi}\right)}{I_{1 / 2}(k)} \phi_{0}-1$,

$\frac{1}{\xi^{2}} \frac{\mathrm{d}}{\mathrm{d} \xi}\left(\xi^{2} \frac{\mathrm{d} \phi_{2}}{\mathrm{~d} \xi}\right)=\left(\frac{6}{\xi^{2}}-\frac{1}{2} \frac{I_{-1 / 2}\left(k \mathrm{e}^{\psi}\right)}{I_{1 / 2}(k)}\right) \phi_{2}$,

with $\phi_{j}=\phi_{j}^{\prime}=0$ at $\xi=0$. In arriving at Eqs. (92) and (93), we have used the identity

$I_{n}^{\prime}(t)=-\frac{n}{t} I_{n-1}(t), \quad(n>0)$

for $n=1 / 2$, which can be easily established from Eq. (87).

We now determine the thermodynamical parameters of a slowly rotating self-gravitating Fermi gas. We can check that the inverse temperature is still given by Eq. (47) and that the relation (46) remains valid. On the other hand, eliminating the central density in Eq. (26), using Eq. (86), we find that

$\eta=\frac{\mu^{2}}{\alpha^{4}} I_{1 / 2}(k)^{2}$,

where

$\mu=\eta_{0} \sqrt{512 \pi^{4} G^{3} M R^{3}}$,

is the "degeneracy parameter" (Chavanis \& Sommeria 1998). Using Eq. (47), we obtain to order $\omega^{2}$ :

$\mu^{2} I_{1 / 2}(k)^{2}=\alpha^{5} \psi^{\prime}(\alpha)\left\{1+2 \omega^{2}\left[\frac{1}{3}+\frac{1}{\alpha} \phi_{0}^{\prime}(\alpha)\right]\right\}$.

This equation relates the uniformizing variable $k$ to the dimensionless box radius $\alpha$. The relation between the angular momentum and the angular velocity is still given by Eq. (53) with the moment of inertia

$\mathcal{I}=\frac{2}{3 \alpha^{4} \psi^{\prime}(\alpha)} \int_{0}^{\alpha} \frac{I_{1 / 2}\left(k \mathrm{e}^{\psi}\right)}{I_{1 / 2}(k)} \xi^{4} \mathrm{~d} \xi$.

Using Eq. (62), the energy can be put in the form

$$
\begin{aligned}
\Lambda= & \frac{\alpha^{7}}{\mu^{4}} \int_{0}^{\alpha}\left\{\frac{I_{3 / 2}\left(k \mathrm{e}^{\psi}\right)}{I_{1 / 2}(k)^{5}}-\frac{3}{2} v \frac{I_{1 / 2}\left(k \mathrm{e}^{\psi}\right)}{I_{1 / 2}(k)^{5}} \phi_{0}(\xi)\right\} \xi^{2} \mathrm{~d} \xi \\
& +\frac{1}{2} \lambda \omega-\frac{2}{3} \frac{\alpha^{10}}{\mu^{4}}\left\{\frac{I_{3 / 2}\left(k \mathrm{e}^{\psi(\alpha)}\right)}{I_{1 / 2}(k)^{5}}-\frac{3}{2} v \frac{I_{1 / 2}\left(k \mathrm{e}^{\psi(\alpha)}\right)}{I_{1 / 2}(k)^{5}} \phi_{0}(\alpha)\right\} .
\end{aligned}
$$

Finally, for the entropy, we obtain (see Appendix A)

$$
\begin{aligned}
\frac{\eta_{0} S}{M}= & \ln k+\eta+\psi(\alpha)-\frac{7}{3} \eta \Lambda-\frac{4}{3} \eta \lambda \omega+v\left[\frac{\alpha^{6}}{6}+\phi_{0}(\alpha)\right] \\
& -\frac{2}{9} \frac{\alpha^{6}}{\mu^{2}}\left\{\frac{I_{3 / 2}\left(k \mathrm{e}^{\psi(\alpha)}\right)}{I_{1 / 2}(k)^{3}}-\frac{3}{2} v \frac{I_{1 / 2}\left(k \mathrm{e}^{\psi(\alpha)}\right)}{I_{1 / 2}(k)^{3}} \phi_{0}(\alpha)\right\} .
\end{aligned}
$$

For $v=0$, Eqs. (47), (97), (99) and (100) reduce to the equations of state derived by Chavanis \& Sommeria (1998) for the non-rotating Fermi gas. For $k \rightarrow+\infty$ (non degenerate limit), we recover the equations derived in Sect. 3 .

The equilibrium phase diagram can be obtained in the following manner. For given $k, \mu$ and $\omega$, we can solve Eqs. (89), (92) and (93) until the value $\xi=\alpha$ at which the relation (97) is satisfied. Then, Eqs. (47) and (99) determine the temperature and the energy of the configuration. If the angular momentum is fixed instead of the angular velocity, we must use Eq. (53) with Eq. (98) to express $\omega$ in terms of $\lambda$. By varying the parameter $k$ (for a fixed value of the degeneracy parameter $\mu$ ), we can cover the whole diagram in parameter space. A complete description of this diagram has been given by Chavanis (2002c) in the non-rotating case.

In Fig. 12, we represent the caloric curve of self-gravitating fermions for a degeneracy parameter $\mu=10^{5}$ and for different values of angular momentum. We observe that degeneracy has the effect of unwinding the spiral of Fig. 5. For sufficiently large values of the degeneracy parameter, there is still gravitational collapse at $\Lambda_{c}$ accompanied by a rise of temperature, but this "gravothermal catastrophe" stops when the core of the system becomes degenerate. This leads to the formation of a "fermion ball" which contains a moderately large fraction of mass $q M$ (at point $D$, we typically have $q \simeq 0.2$ ). In the microcanonical ensemble, the decrease of potential energy in the core is compensated by an increase of temperature. Therefore, the mass $(1-q) M$ contained in the halo undergoes an expansion which, in our model, is arrested by the walls of the box. As a result, the density of the halo is almost uniform. Typical density profiles are given by Chavanis \& Sommeria (1998) in the non rotating case. Because of the expansion of the halo, the moment of inertia of the system increases during the collapse despite the formation of a massive nucleus. Therefore, the angular velocity decreases contrary to what might have been expected. The angular velocity is represented as a function of energy in Fig. 13. It has a complicated behaviour which corresponds to the unwindement of the spiral of Fig. 6.

For smaller values of the degeneracy parameter, the gravitational phase transition is suppressed (Chavanis \& Sommeria 1998; Chavanis 2002c) and the caloric curve has the structure of Fig. 14. This diagram is similar to the one found by Fliegans \& Gross (2002) in their two-dimensional model of rotating self-gravitating systems with a relatively large cutoff radius (which plays the role of the inverse of our degeneracy parameter). Indeed, when the cut-off radius (or degeneracy) is sufficiently large, the spiral of Fig. 12 unwinds and the $\eta(\Lambda)$ curve is univalued like in Fig. 14. For high energies, the density is homogeneous. For low energies, the equilibrium states have a core-halo structure with a partially degenerate nucleus and a dilute envelope. As energy decreases any further, 


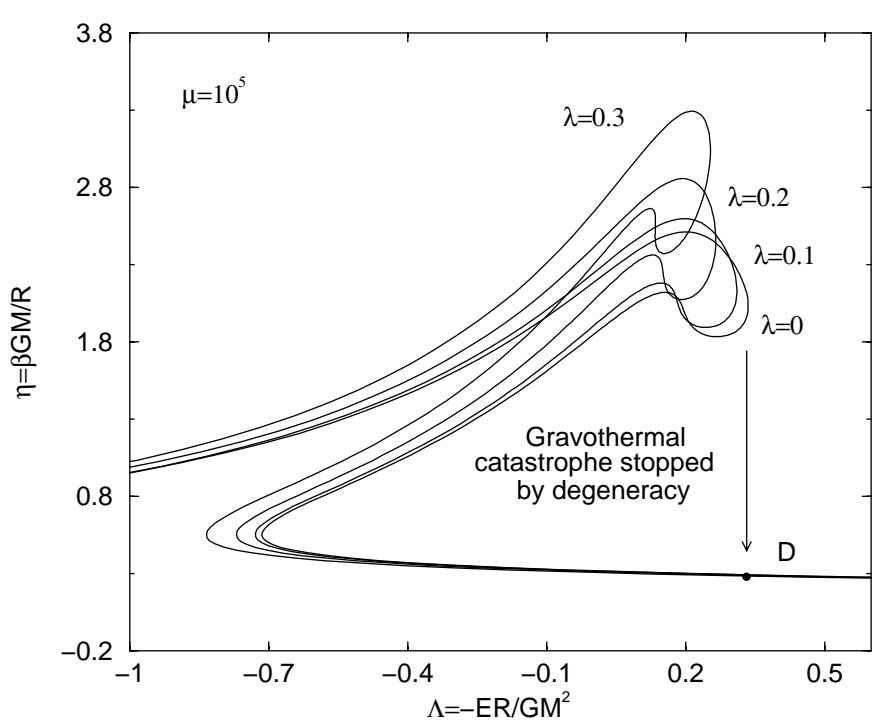

Fig. 12. Equilibrium phase diagram of self-gravitating fermions giving the inverse temperature $\eta$ as a function of minus the energy $\Lambda$ for different values of the angular momentum $\lambda$ and for a degeneracy parameter $\mu=10^{5}$.

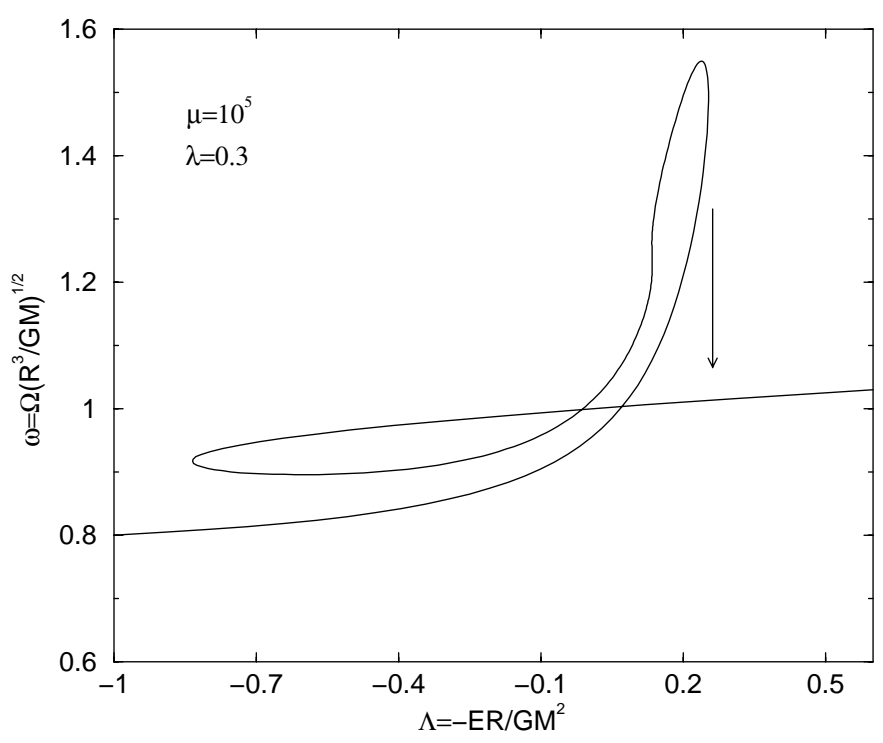

Fig. 13. Angular velocity vs energy for self-gravitating fermions with a degeneracy parameter $\mu=10^{5}$ and an angular momentum $\lambda=0.3$.

the nucleus contains more and more mass and becomes smaller and smaller. This is a property of the $R \sim M^{-1 / 3}$ law of degenerate configurations (like white dwarfs). For rotating systems described in the microcanonical ensemble (fixed $E$ and $L$ ), the resulting decrease of moment of inertia is accompanied by an increase of angular velocity as shown in Fig. 15. In this diagram, the spiral of Fig. 6 is completely unwound and the angular velocity increases monotonically with $\Lambda$.

In Fig. 16, we have focused our attention to what happens close to the minimum energy $E_{\min }$ corresponding to $T=0$. At that point, the system has the same structure as a cold white dwarf star (see Appendix B). For our perturbative analysis to be valid even after collapse, we have taken a very small angular momentum $\lambda=0.01$. We shall describe the diagram of

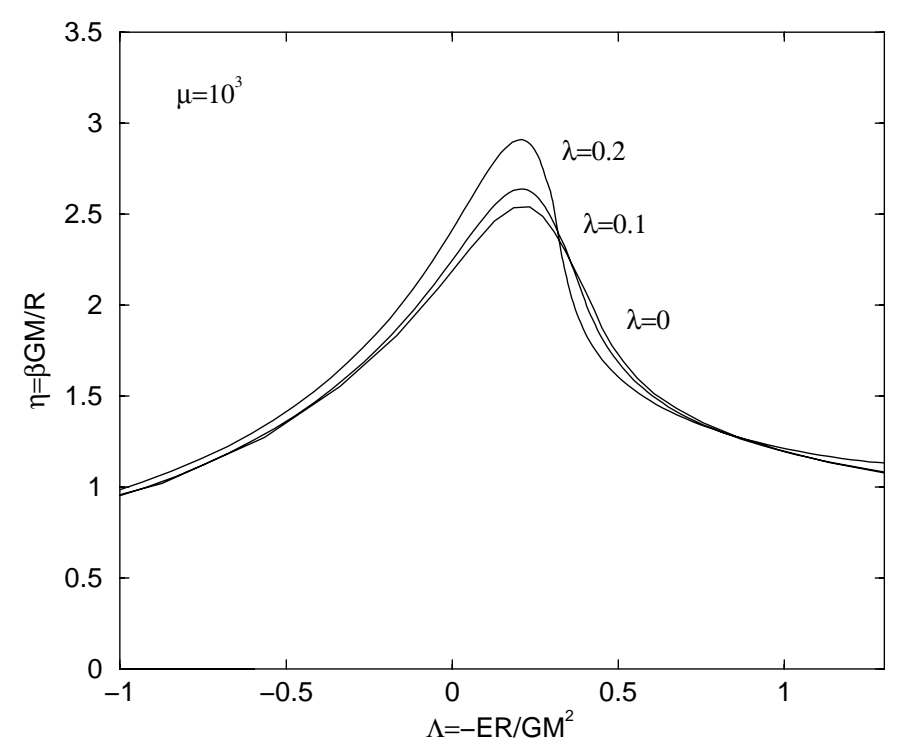

Fig. 14. Same as Fig. 12 for $\mu=10^{3}$. For high degeneracy, the spiral unwinds and the gravitational phase transition in the microcanonical ensemble is suppressed.

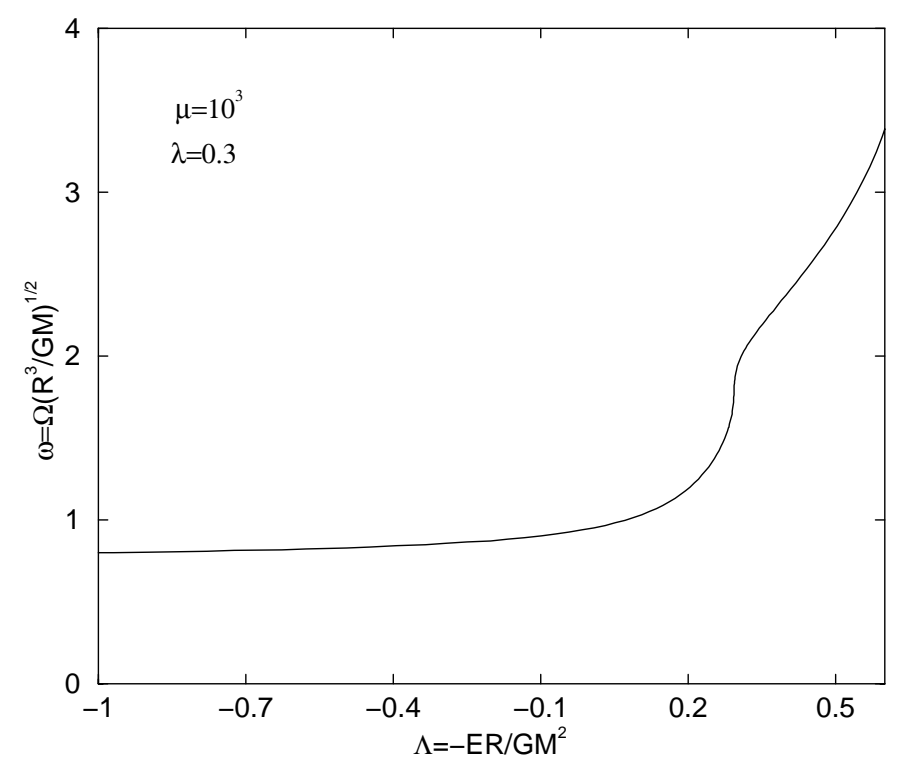

Fig. 15. Same as Fig. 13 for $\mu=10^{3}$.

Fig. 16 in the $(L, T)$ ensemble in which the angular momentum and the temperature are fixed (this is a situation intermediate between microcanonical and canonical ensembles). For $T>T_{\mathrm{c}}$, the system is in a gaseous phase with an almost homogeneous density profile. For $T<T_{\mathrm{c}}$, the system undergoes an isothermal collapse that only stops when gravity is balanced by the degeneracy pressure. The result of this phase transition is a "fermion ball" which contains almost all the mass $(q \simeq 1)$ unlike in the microcanonical ensemble at the point of gravothermal catastrophe. These fermion balls can be of astrophysical interest in Dark Matter models consisting of massive neutrinos (Bilic \& Viollier 1997; Chavanis 2002e). For rotating systems, the isothermal collapse is accompanied by a discontinuous rise of angular velocity at the critical temperature $T_{\mathrm{c}}$ (see Fig. 17). Therefore, even if the initial rotation of the system is negligible 


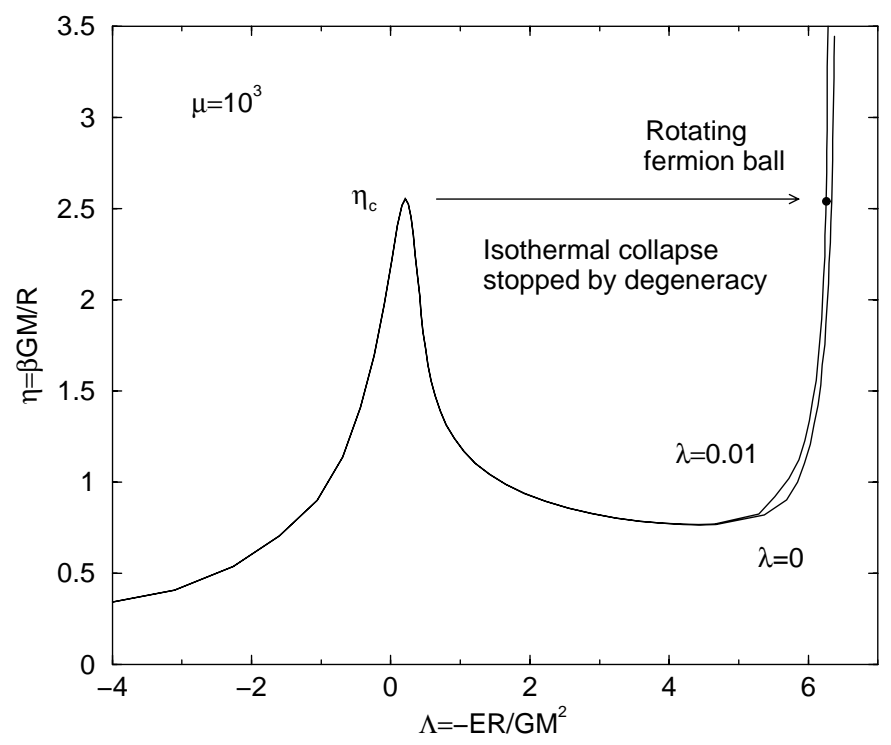

Fig. 16. Equilibrium phase diagram for self-gravitating fermions with a degeneracy parameter $\mu=10^{3}$ and an angular momentum $\lambda=0.01$. For $T<T_{\mathrm{c}}$, i.e., $\eta>\eta_{\mathrm{c}}$, the system undergoes an isothermal collapse leading to a rotating fermion ball containing a large fraction of mass and angular momentum.

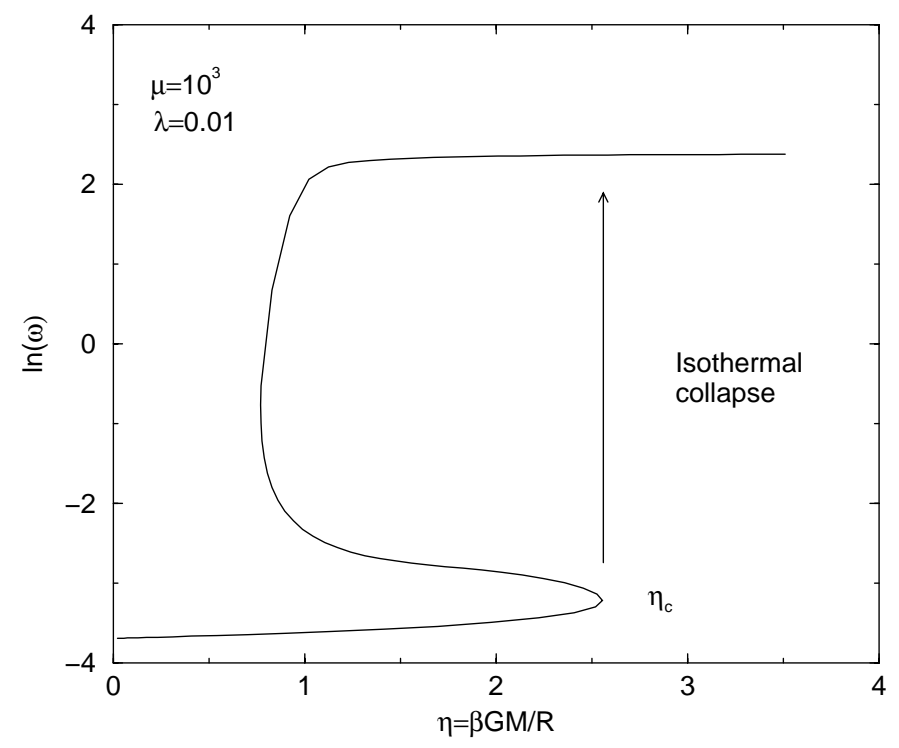

Fig. 17. Angular velocity vs. temperature plot for $\mu=10^{3}$ and $\lambda=0.01$. The gravitational collapse below $T_{\mathrm{c}}$ is accompanied by a discontinuous jump of angular velocity due to the moment of inertia decrease. The resulting fermion ball rotates with angular velocity $\Omega \simeq 0.1891 G^{1 / 2} M^{1 / 2} R_{0}^{-3 / 2}$ (see Appendix B) corresponding to an increase $\sim 270$ of the initial angular velocity. The dimensionless rotation rate $v \simeq 3.98 \times 10^{-3}$ is sufficiently small to justify our perturbative approach.

in the gaseous phase, after collapse the "fermion ball" can have appreciable rotation as suggested in Fig. 17. Its structure is then similar to a distorted polytrope of index $n=3 / 2$ as computed by Chandrasekhar (1933) in the limit of slow rotation.

\section{Conclusion}

In this paper, we have considered the effect of a small rotation on the thermodynamic stability of self-gravitating systems. We have worked in a finite spherical box in order to make a clear connexion with the Antonov problem for non-rotating systems and render the statistical mechanics of these objects rigorous. Physically, this idealization means that our isothermal system is surrounded by a medium which fixes its size. We have found that for rotating systems, the well-known inequivalence of statistical ensembles for self-gravitating systems manifests itself in a striking manner: the instability is advanced in the microcanonical ensemble and delayed in the canonical one. In addition, we have found a connexion between the onset of instability and the configuration of maximum flattening in the series of equilibria. These results have been generalized to the case of self-gravitating fermions.

On the other hand, the case of rapidly rotating isothermal configurations is interesting because new, non trivial, structures can emerge as maximum entropy states (i.e., most probable states). The classification of such structures is complicated because many bifurcations can occur depending on the values of the control parameters $(M, E, L)$. In particular, we must be careful to select only entropy maxima, discarding the critical points of entropy which are only saddle points. This can be obtained either by solving the Boltzmann-Poisson equation (Votyakov et al. 2002) and checking the stability of the solutions or by using relaxation equations towards the maximum entropy state (Chavanis et al. 1996). These studies are important in order to obtain a classification of the most probable configurations of self-gravitating systems. Such a classification scheme is also relevant in the very similar context of two-dimensional vortices described as maximum entropy structures (e.g., Chavanis \& Sommeria 1996, 1998). The idea of this classification was proposed in an earlier paper (Chavanis et al. 1996) and will be given further attention elsewhere (Chavanis \& Rieutord 2002). We must be aware, however, that the statistical approach is conditioned by an ergodicity hypothesis which may not be completely fulfilled in practice. This complicated problem of incomplete relaxation demands further discussion.

Acknowledgements. I acknowledge interesting discussions with J. Katz. I have also benefited from stimulating interactions with the participants of the Les Houches School of Physics on "Dynamics and thermodynamics of systems with long range interactions" (February 2002).

\section{Appendix A: The entropy of the rotating self-gravitating Fermi gas}

The Fermi-Dirac entropy (85) can be written in the equivalent form

$S=-\int\left\{\frac{f}{\eta_{0}} \ln \left(\frac{f / \eta_{0}}{1-f / \eta_{0}}\right)+\ln \left(1-\frac{f}{\eta_{0}}\right)\right\} \mathrm{d}^{3} \boldsymbol{r} \mathrm{d}^{3} \boldsymbol{v}$

Inserting the distribution function

$f=\frac{\eta_{0}}{1+k \mathrm{e}^{\Psi} \mathrm{e}^{\beta \frac{\omega^{2}}{2}}}$, 
deduced from Eq. (84) in Eq. (A.1), we obtain

$$
\begin{aligned}
\eta_{0} S= & M \ln k+\int \rho \Psi \mathrm{d}^{3} \boldsymbol{r}+\beta K_{\text {th }} \\
& +\eta_{0} \int \ln \left(1+\frac{1}{k} \mathrm{e}^{-\Psi} \mathrm{e}^{-\beta \frac{w^{2}}{2}}\right) \mathrm{d}^{3} \boldsymbol{r} \mathrm{d}^{3} \boldsymbol{v} .
\end{aligned}
$$

The last integral can be reduced by an integration by parts to the expression $(2 / 3) \beta K_{\text {th }}$. Therefore,

$\eta_{0} S=M \ln k+\int \rho \Psi \mathrm{d}^{3} \boldsymbol{r}+\frac{5}{3} \beta K_{\mathrm{th}}$.

Now, using the definition of $\Psi$, we get

$\eta_{0} S=M \ln k+2 \beta W-\beta K_{\mathrm{rot}}-M \beta \Phi_{0}+\frac{5}{3} \beta K_{\mathrm{th}}$.

Since $W=E-K$ and $K_{\mathrm{th}}=K-K_{\text {rot }}$, the foregoing expression can be rewritten

$\eta_{0} S=M \ln k+2 \beta E-\frac{1}{3} \beta K-M \beta \Phi_{0}-\frac{8}{3} \beta K_{\mathrm{rot}}$,

or, according to Eqs. (59) and (62),

$\eta_{0} S=M \ln k+\frac{7}{3} \beta E-\frac{1}{3} \beta \oint p \boldsymbol{r} \mathrm{d} \boldsymbol{S}-M \beta \Phi_{0}-\frac{4}{3} \beta \boldsymbol{L} \cdot \mathbf{\Omega}$.

The pressure at the surface of the sphere can be calculated with Eqs. (56) and (A.2). After simplification, we get

$$
\begin{aligned}
\frac{\eta_{0} S}{M}= & \ln k-\frac{7}{3} \eta \Lambda-\beta \Phi_{0}-\frac{4}{3} \eta \lambda \omega \\
& -\frac{2}{9} \eta \frac{\alpha^{10}}{\mu^{4}}\left\{\frac{I_{3 / 2}\left(k \mathrm{e}^{\psi(\alpha)}\right)}{I_{1 / 2}(k)^{5}}-\frac{3}{2} v \frac{I_{1 / 2}\left(k \mathrm{e}^{\psi(\alpha)}\right)}{I_{1 / 2}(k)^{5}} \phi_{0}(\alpha)\right\} .
\end{aligned}
$$

Using Eq. (71), which remains valid for self-gravitating fermions, and Eq. (95) we finally arrive at the form (100) for the entropy.

\section{Appendix B: The energy of a slowly rotating polytrope}

In this Appendix, we derive a simple analytic formula for the potential energy of a slowly rotating polytrope of index $n$. The index $n=3 / 2$ describes a completely degenerate Fermi gas at zero temperature, which is a particular limit of the model studied in Sect. 5.

For a self-gravitating system rotating with constant angular velocity $\Omega$, the condition of hydrostatic equilibrium in the rotating frame can be written

$\nabla p=-\rho \nabla \Phi_{\mathrm{eff}}$,

where $\Phi_{\text {eff }}$ is the effective potential defined in Eq. (11). Now, for a polytropic equation of state

$p=K \rho^{1+\frac{1}{n}}$,

the equation of hydrostatic equilibrium can be rewritten as

$(n+1) \nabla\left(\frac{p}{\rho}\right)=-\nabla \Phi_{\text {eff }}$
This equation integrates to give

$$
(n+1) p=\rho\left(\Phi_{\mathrm{eff}}^{b}-\Phi+\frac{1}{2}|\boldsymbol{\Omega} \times \boldsymbol{r}|^{2}\right),
$$

where $\Phi_{\mathrm{eff}}^{b}$ is the constant value of the effective potential at the surface of the polytrope. On integrating Eq. (B.4) over the volume of the configuration, we obtain

$(n+1) \int p \mathrm{~d}^{3} \boldsymbol{r}=M \Phi_{\mathrm{eff}}^{b}-2 W+\frac{1}{2} \boldsymbol{L} \cdot \boldsymbol{\Omega}$.

Inserting this last relation in Eq. (57), and recalling that the pressure vanishes on the surface of a polytrope, we get

$W=\frac{1}{5-n}\left[3 M \Phi_{\mathrm{eff}}^{b}+\left(n+\frac{5}{2}\right) \boldsymbol{L} \cdot \boldsymbol{\Omega}\right]$.

This general expression was derived by Chandrasekhar (1961) in a slightly different manner. We now consider the case of slowly rotating polytropes. In that case, an explicit expression for $\Phi_{\mathrm{eff}}^{b}$ can be deduced from the study of Chandrasekhar (1933) on distorted polytropes. The density profile can be written

$\rho=\rho_{0} \Theta^{n}$,

with

$\Theta=\theta+v\left\{\psi_{0}(\xi)+\sum_{j=1}^{+\infty} A_{j} \psi_{j}(\xi) P_{j}(\mu)\right\}$.

The quantities have their usual meaning: $\rho_{0}$ is the central density, $v=\Omega^{2} / 2 \pi G \rho_{0}, \theta$ is the solution of the Lane-Emden equation of index $n$ and $P_{j}(\mu)$ are Legendre polynomials. The functions $\psi_{j}(\xi)$ have been defined and tabulated by Chandrasekhar (1933). The inner and outer potentials are given by

$\Phi_{\text {int }}=\Phi_{\mathrm{eff}}^{b}-R\left[\Theta-\frac{1}{6} v \xi^{2}\left(1-P_{2}(\mu)\right)\right]$

$\Phi_{\mathrm{ext}}=-R\left[\frac{C_{0}}{\xi}+v \sum_{j=1}^{+\infty} \frac{C_{j}}{\xi^{j+1}} P_{j}(\mu)\right]$,

with $R \equiv(n+1) K \rho_{0}^{1 / n}$. The constant $A_{j}, C_{j}$ are determined by requiring the continuity of $\Phi$ and its derivative on a sphere of radius $\xi_{1}$, the first zero of the Lane-Emden function with index $n$. For our purposes, we just need to consider the equations obtained for $j=0$ :

$R \frac{C_{0}}{\xi_{1}}=R v \psi_{0}\left(\xi_{1}\right)-\frac{1}{6} R v \xi_{1}^{2}-\Phi_{\mathrm{eff}}^{b}$,

$-R \frac{C_{0}}{\xi_{1}^{2}}=R \theta_{1}^{\prime}+R v \psi_{0}^{\prime}\left(\xi_{1}\right)-\frac{1}{3} R v \xi_{1}$.

In arriving at Eq. (B.11), we have used $\theta\left(\xi_{1}\right)=0$. Now, the relation between the mass and the central density is given by (Chandrasekhar 1933, Eq. (40)):

$M=-4 \pi\left[\frac{(n+1) K}{4 \pi G} \rho_{0}^{\frac{3-n}{3 n}}\right]^{3 / 2} \xi_{1}^{2} \theta_{1}^{\prime}\left[1+v \frac{\frac{1}{3} \xi_{1}-\psi_{0}^{\prime}\left(\xi_{1}\right)}{\left|\theta_{1}^{\prime}\right|}\right]$. 
On the other hand, we define the characteristic radius $R_{\Omega}$ of a rotating polytrope by the relation (see Chandrasekhar 1933, Eq. (9)):

$R_{\Omega}=\left[\frac{(n+1) K}{4 \pi G} \rho_{0}^{\frac{1}{n}-1}\right]^{1 / 2} \xi_{1}$.

Inserting Eq. (B.12) in Eq. (B.13) and using Eq. (B.14), we obtain

$R \frac{C_{0}}{\xi_{1}}=\frac{G M}{R_{\Omega}}$

On the other hand, we can check that

$R v=\frac{2 \Omega^{2}}{\xi_{1}^{2}} R_{\Omega}^{2}$.

Substituting these results in Eq. (B.11), we find that the value of the effective potential at the surface of a slowly rotating polytrope is given by

$\Phi_{\mathrm{eff}}^{b}=-\frac{G M}{R_{\Omega}}+2 \Omega^{2} R_{\Omega}^{2}\left(\frac{\psi_{0}\left(\xi_{1}\right)}{\xi_{1}^{2}}-\frac{1}{6}\right)$.

We now need to relate the characteristic radius $R_{\Omega}$ to the total mass $M$ of the system. Eliminating the central density $\rho_{0}$ between Eq. (B.13) and Eq. (B.14), and expressing $v$ in terms of $R_{\Omega}$ with the aid of Eq. (B.16), we obtain to order $\Omega^{2}$ :

$R_{\Omega}=R_{0}\left[1+\Omega^{2} \frac{S_{n} K^{\frac{3 n}{3-n}}}{2 \pi G^{\frac{2 n+3}{3-n}} M^{\frac{2 n}{3-n}}}\right]$

where $R_{0}$ is the radius of a non-rotating polytrope. It is related to its mass $M$ by the relation

$G N_{n} M^{\frac{n-1}{n}} R_{0}^{\frac{3-n}{n}}=K$

The constant $N_{n}$ is defined and tabulated in Chandrasekhar (1942) for different values of the polytropic index $n$. The constant $S_{n}$ appearing in Eq. (B.18) is given by

$S_{n}=\frac{n-1}{3-n}\left(\frac{n+1}{4 \pi}\right)^{\frac{n}{1-n}} N_{n}^{\frac{2 n^{2}}{(1-n)(3-n)}} \xi_{1}^{\frac{2 n}{1-n}} \frac{\frac{1}{3} \xi_{1}-\psi_{0}^{\prime}\left(\xi_{1}\right)}{\left|\theta_{1}^{\prime}\right|}$,

and typical values are listed in Table B.1.

From the above results, we can obtain an explicit expression for the potential energy of a slowly rotating polytrope. For an axisymmetric system, we have the relation $\boldsymbol{L}=I \mathbf{\Omega}$ where $I$ is the axial moment of inertia (51). To our order of approximation, we just need to determine the value of $I$ for a non-rotating polytrope. Using the relations $\rho=\rho_{0} \theta^{n}$ and $r=\xi R_{0} / \xi_{1}$, expressing the central density as a function of $R_{0}$ by the relation (B.14) and using the mass-radius relation (B.19), we obtain

$I=\mathcal{I}_{n} M R_{0}^{2}$,

with

$\mathcal{I}_{n}=\frac{8 \pi}{3}\left[\frac{4 \pi}{(n+1) N_{n}}\right]^{\frac{n}{1-n}} \xi_{1}^{\frac{5-3 n}{n-1}} \int_{0}^{\xi_{1}} \theta^{n} \xi^{4} \mathrm{~d} \xi$
Table B.1. The constants of slowly rotating polytropes.

\begin{tabular}{llllll}
\hline \hline$n$ & $N_{n}$ & $S_{n}$ & $\mathcal{I}_{n}$ & $Q_{n}$ & $Z_{n}$ \\
\hline 1 & 0.63662 & 0 & 0.08320 & -0.1009 & 0.20264 \\
1.5 & 0.42422 & 8.73193 & 0.20461 & 0.40008 & 0.11127 \\
2 & 0.36475 & 557.60 & 0.15485 & 0.30331 & 0.05846 \\
3 & 0.36394 & $\infty$ & 0.07536 & 0.15087 & 0.01230 \\
4 & 0.47720 & $-3.8 \times 10^{-5}$ & 0.02257 & 0.04434 & 0.00107 \\
\hline
\end{tabular}

Therefore, according to Eqs. (B.6), (B.17) and (B.21), the potential energy of a slowly rotating polytrope of index $n$ is given by

$W=\frac{1}{5-n}\left(-\frac{3 G M^{2}}{R_{\Omega}}+Q_{n} M R_{0}^{2} \Omega^{2}\right)$,

with

$Q_{n}=6 \frac{\psi_{0}\left(\xi_{1}\right)}{\xi_{1}^{2}}-1+\left(n+\frac{5}{2}\right) \mathcal{I}_{n}$

where $R_{0}$ and $R_{\Omega}$ are given by Eqs. (B.19) and (B.18) respectively. For $\Omega=0$, Eq. (B.23) reduces to Ritter's formula (see Chandrasekhar 1942). Note also that the dimensionless rotation parameter can be written

$v=Z_{n} \frac{\Omega^{2} R_{0}^{3}}{G M}$,

with

$Z_{n}=\frac{1}{2 \pi}\left(\frac{n+1}{4 \pi}\right)^{\frac{n}{1-n}} N_{n}^{\frac{n}{1-n}} \xi_{1}^{\frac{2 n}{1-n}}$.

The polytropic indices $n=1$ and $n=3$ are special. For $n=1$, the problem can be solved analytically. The following results are well-known (Chandrasekhar 1942, 1933): $\xi_{1}=\pi$, $\theta_{1}^{\prime}=-1 / \pi, \psi_{0}\left(\xi_{1}\right)=1, \psi_{0}^{\prime}\left(\xi_{1}\right)=1 / \pi, N_{1}=2 / \pi$. According to Eq. (B.14), the radius $R_{\Omega}=R_{0}=(K / 2 \pi G)^{1 / 2} \xi_{1}$ is independant of the central density and is uniquely determined by the value of $K$. The mass $M$ is independant of the radius. Equations (B.21) and (B.25) remain valid with $\mathcal{I}_{1}=2\left(\pi^{2}-\right.$ $6) / 3 \pi^{3}$ and $Z_{1}=2 / \pi^{2}$. The other quantities can be obtained from these results (see Table B.1). The case $n=3$ corresponds to the reverse situation. For $\Omega=0$, the mass $M_{0}=\left(K / G N_{3}\right)^{3 / 2}$ is independant of the central density and is uniquely determined by the value of $K$. The radius is independant of the mass. This mathematical property is related to the limiting mass of relativistic white dwarfs (Chandrasekhar 1942). For $\Omega \neq 0$, the mass-radius relation (B.18) can be written

$M=M_{0}\left[1+\Omega^{2} S_{3}^{\prime} \frac{G^{1 / 2} R_{\Omega}^{3}}{2 K^{3 / 2}}\right]$,

with

$S_{3}^{\prime}=\frac{\pi^{1 / 2}}{\xi_{1}^{3}} \frac{\frac{1}{3} \xi_{1}-\psi_{0}^{\prime}\left(\xi_{1}\right)}{\left|\theta_{1}^{\prime}\right|} \simeq 0.03309$. 
We now consider the case of a non relativistic degenerate Fermi gas at zero temperature. As is well-known, this system is equivalent to a polytrope of index $n=3 / 2$. In addition, the constant $K$ is explicitly given by

$K=\frac{1}{5}\left(\frac{3}{4 \pi \eta_{0}}\right)^{2 / 3}$,

where $\eta_{0}$ is the phase space density of a completely degenerate Fermi gas. According to the Virial theorem (62), the total energy $E=K+W$ of this system is $E=\frac{W}{2}$. Therefore, using Eq. (B.23), we have

$E=-\frac{3 G M^{2}}{7 R_{\Omega}}+\frac{1}{7} Q_{3 / 2} M R_{0}^{2} \Omega^{2}$.

According to Eq. (B.19), the mass-radius relation of a non rotating "fermion ball" is

$M R_{0}^{3}=\frac{\chi}{\eta_{0}^{2} G^{3}}$,

with

$\chi \equiv \frac{1}{125}\left(\frac{3}{4 \pi}\right)^{2} \frac{1}{N_{3 / 2}^{3}} \simeq 5.9723 \times 10^{-3}$.

For a rotating fermion ball we have, according to Eq. (B.18),

$R_{\Omega}=R_{0}\left(1+\frac{\kappa \Omega^{2}}{2 \pi G^{4} M^{2} \eta_{0}^{2}}\right)$,

with

$\kappa \equiv \frac{1}{125}\left(\frac{3}{4 \pi}\right)^{2} S_{3 / 2} \simeq 3.9813 \times 10^{-3}$.

Then, the energy (B.30) can be expressed in terms of $M, G, \eta_{0}$ and $\Omega$. In order to make the link with the variables introduced in Sect. 5, which are normalized by the box radius $R$, we note the relation

$\frac{R_{0}}{R}=\left(512 \pi^{4} \chi\right)^{1 / 3} \mu^{-2 / 3}=\frac{6.6784}{\mu^{2 / 3}}$,

which directly results from Eq. (B.31) and the definition (96) of the degeneracy parameter $\mu$.

\section{References}

Antonov, V. A. 1962, Vest. Leningr. Gos. Univ., 7, 135

Bilic, N., \& Viollier, R. D. 1997, Phys. Lett. B, 408, 75
Binney, J., Tremaine, S. 1987, Galactic Dynamics (Princeton Series in Astrophysics)

Cerruti-Sola, M., Cipriani, P., \& Pettini, M. 2001, MNRAS, 328, 339

Chandrasekhar, S. 1933, MNRAS, 93, 390

Chandrasekhar, S. 1942, An Introduction to the Theory of Stellar Structure (Dover)

Chandrasekhar, S. 1961, ApJ, 134, 662

Chavanis, P. H. 2002a, A\&A, 381, 340

Chavanis, P. H. 2002b, A\&A, 381, 709

Chavanis, P. H. 2002c, PRE, 65, 056123

Chavanis, P. H. 2002d, A\&A, 386, 732

Chavanis, P. H. 2002e, in Proc. of the Fourth International Heidelberg Conf. on Dark Matter in Astro and Particle Physics, edited by Klapdor-Kleingrothaus, H.V. (Springer, New-York) [astro-ph/0205426]

Chavanis, P. H., \& Rieutord, M. 2002, in preparation

Chavanis, P. H., \& Sommeria, J. 1996, J. Fluid. Mech., 314, 267

Chavanis, P. H., \& Sommeria, J. 1998, J. Fluid. Mech., 356, 259

Chavanis, P. H., \& Sommeria, J. 1998, MNRAS, 296, 569

Chavanis, P. H., Sommeria, J., \& Robert, R. 1996, ApJ, 471, 385

Chavanis, P. H., Rosier, C., \& Sire, C. 2002, PRE, 66, 036105

de Vega, H. J., Sanchez, N., \& Combes, F. 1998, ApJ, 500, 8

de Vega H. J., Sanchez, N. 2002, Nucl. Phys. B, 625, 409

Fliegans, O., \& Gross, D. H. E. 2002, PRE, 65, 046143

Follana, E., \& Laliena, V. 2000, PRE, 61, 6270

Gross, D. H. E. 2001, Microcanonical thermodynamics: Phase transitions in "Small" systems (World Scientific, Singapore)

Hertel, P., \& Thirring, W. 1971, Commun. Math. Phys., 24, 22

Huber, D., \& Pfenniger, D. 2002, A\&A, 386, 359

Horwitz, G., \& Katz, J. 1978, ApJ, 222, 941

Ispolatov, I., \& Cohen, E. G. D. 2001, PRE, 64, 6103

Katz, J. 1978, MNRAS, 183, 765

Lagoute, C., \& Longaretti, P. Y. 1996, A\&A, 308, 441

Laliena, V. 1999, PRE, 59, 4786

Lynden-Bell, D. 1967, MNRAS, 136, 101

Lynden-Bell, D. 2000, unpublished [astro-ph/0007116]

Lynden-Bell, D., \& Wood, R. 1968, MNRAS, 138, 495

Milne, E. A. 1923, MNRAS, 83, 118

Padmanabhan, T. 1989, ApJS, 71, 651

Padmanabhan, T. 1990, Phys. Rep., 188, 285

Semelin, B., Sanchez, N., \& de Vega, H. J. 2001, Phys. Rev. D, 63, 084005

Sire, C., \& Chavanis, P. H. 2002, PRE, 66, 046133

Taruya, A., \& Sakagami, M. 2002, Physica A, 307, 185

Tremaine, S., Hénon, M., \& Lynden-Bell, D. 1987, MNRAS, 227, 543

van Albada, T. S. 1982, MNRAS, 201, 939

Votyakov, E. V., Hidmi, H. I., De Martino, A., \& Gross, D. H. E. 2002, PRL, 89, 031101

Youngkins, V. P., \& Miller, B. N. 2000, PRE, 62, 4582 